\title{
On Fano varieties whose effective divisors are numerically eventually free
}

\author{
StÉPhANE DRUEL
}

In this paper we classify mildly singular Fano varieties with maximal Picard number whose effective divisors are numerically eventually free. In addition, we prove that if a Del Pezzo surface of degree $r$ admits a finite morphism of degree $>1$ onto a Del Pezzo surface of degree $s$, then either $r=s \geqslant 6$, or $r<s$ and $s \geqslant 8$.

\section{Introduction}

Let $X$ be a normal projective variety and consider the finite dimensional dual $\mathbb{R}$-vector spaces

$$
\mathrm{N}_{1}(X)=(\{1-\text { cycles }\} / \equiv) \otimes \mathbb{R} \quad \text { and } \quad \mathrm{N}^{1}(X)=(\operatorname{Pic}(X) / \equiv) \otimes \mathbb{R}
$$

where $\equiv$ denotes numerical equivalence. The dimension $\rho(X)=\operatorname{dim} \mathrm{N}^{1}(X)=$ $\operatorname{dim} \mathrm{N}_{1}(X)$ is called the Picard Number of $X$. The Mori cone of $X$ is the closure $\overline{\mathrm{NE}}(X) \subset \mathrm{N}_{1}(X)$ of the cone $\mathrm{NE}(X)$ spanned by classes of effective curves. Its dual cone is the nef cone $\operatorname{Nef}(X) \subset \mathrm{N}^{1}(X)$, which by Kleiman's criterion is the closure of the cone spanned by ample classes. The closure of the cone spanned by effective classes in $\mathrm{N}^{1}(X)$ is the pseudo-effective cone $\operatorname{Psef}(X)$. These cones

$$
\operatorname{Nef}(X) \subset \operatorname{Psef}(X) \subset \mathrm{N}^{1}(X)
$$

carry geometric information about the variety $X$. It is natural to try to describe (normal projective) varieties $X$ with $\operatorname{Nef}(X)=\operatorname{Psef}(X)$. The simplest examples of complex projective manifolds with $\operatorname{Nef}(X)=\operatorname{Psef}(X)$ are given by manifolds with Picard number 1 and homogeneous spaces. The case of projective space bundles over curves was worked out by Fulger in [13]. If $\mathscr{E}$ is a locally free sheaf of finite rank on a smooth complex projective curve $C$, then $\operatorname{Nef}\left(\mathbb{P}_{C}(\mathscr{E})\right)=\operatorname{Psef}\left(\mathbb{P}_{C}(\mathscr{E})\right)$ if and only if $\mathscr{E}$ is 
semistable (see [13, Lemma 3.2]). A smooth projective toric variety $X$ satisfies $\operatorname{Nef}(X)=\operatorname{Psef}(X)$ if and only if $X$ is isomorphic to a product of projective spaces by $[12$, Proposition 5.3]. The case of smooth projective horospherical varieties is addressed in [21].

Let $X$ be a normal projective variety such that $K_{X}$ is $\mathbb{Q}$-Cartier. We say that $X$ is $\mathbb{Q}$-Fano if $-K_{X}$ is ample. The Mori cone of a $\mathbb{Q}$-Fano variety with $\log$ canonical singularities is rational polyhedral, and generated by classes of curves (see [10, Theorem 16.6]). The geometry of $X$ is reflected to a large extent in the combinatorial properties of $\mathrm{NE}(X)=\overline{\mathrm{NE}}(X)$. Every face $V$ of $\mathrm{NE}(X)$ corresponds to a surjective morphism with connected fibers $\varphi: X \rightarrow$ $Y$ onto a normal projective variety, which is called a Mori contraction. The morphism $\varphi$ contracts precisely those curves on $X$ with class in $V$ (see [10, Theorem 16.4]). Conversely, any morphism with connected fibers onto a normal projective variety arises in this way.

In this paper we address mildly singular $\mathbb{Q}$-Fano varieties with $\operatorname{Nef}(X)=$ $\operatorname{Psef}(X)$. The main theorem of this paper is the following.

Theorem 1.1. Let $X$ be a $\mathbb{Q}$-Fano variety with locally factorial canonical singularities. Suppose that $\operatorname{Nef}(X)=\operatorname{Psef}(X)$. Then $\rho(X) \leqslant \operatorname{dim} X$, and equality holds only if $X \cong X_{1} \times \cdots \times X_{m}$ where $X_{i}$ is a double cover of $\mathbb{P}^{1} \times \cdots \times \mathbb{P}^{1}$ branched along a reduced divisor of type $(2, \ldots, 2)$ for each $i \in\{1, \ldots, m\}$.

Remark 1.2. Example 9.2 shows that the statement of Theorem 1.1 does not hold for $\mathbb{Q}$-Fano varieties with Gorenstein canonical singularities.

Occhetta, Solá Conde, Watanabe, and Wiśniewski recently posted a somewhat related result. They proved in [24] that a Fano manifold whose elementary contractions are $\mathbb{P}^{1}$-fibrations is rational homogenous, without any assumption on the Picard number of $X$ (see also [5, Proposition 2.4]).

The argument for the proof of Theorem 1.1 goes as follows. Suppose furthermore that $\operatorname{dim} X \geqslant 3$. The first step in the proof of Theorem 1.1 is to show that the Mori cone $\mathrm{NE}(X)$ is simplicial. This is done in Lemma 4.9. Then we argue by induction on the dimension of $X$. If $X \rightarrow Y$ is a contraction, then it is easy to see that $Y$ satisfies all the conditions listed in Theorem 1.1.

Suppose first that there is a contraction $X \rightarrow Y$ where $Y$ is a double cover of $\mathbb{P}^{1} \times \cdots \times \mathbb{P}^{1}$ branched along a divisor of type $(2, \ldots, 2)$. It is easy to see that we must have $\operatorname{dim} Y \geqslant 3$ (see Proposition 6.1) and that there 
is a contraction $X \rightarrow T$ such that the induced morphism $X \rightarrow Y \times T$ is surjective and finite. We conclude that $X \cong Y \times T$ using the following result.

Theorem 1.3. Let $Z$ be a $\mathbb{Q}$-Fano variety of dimension $\geqslant 3$ with Gorenstein canonical singularities, $Y$ a double cover of $\mathbb{P}^{1} \times \cdots \times \mathbb{P}^{1}$ branched along a reduced divisor $B=\sum_{j \in J} B_{j}$ of type $(2, \ldots, 2)$, and $f: Z \rightarrow Y$ a finite morphism. Suppose that $B_{j}$ is ample for each $j \in J$. Then $\operatorname{deg}(f)=1$.

Suppose now that for each contraction $X \rightarrow Y$, we have $Y \cong \mathbb{P}^{1} \times \cdots \times$ $\mathbb{P}^{1}$. Then apply the following characterization result to complete the proof of Theorem 1.1.

Proposition 1.4. Let $X$ be a $\mathbb{Q}$-Fano variety of dimension $\geqslant 3$ with Gorenstein canonical singularities. Suppose that $\operatorname{Nef}(X)=\operatorname{Psef}(X)$ and $\rho(X)=$ $\operatorname{dim} X$. Suppose furthermore that each non-trivial contraction $X \rightarrow Y$ with $\operatorname{dim} Y<\operatorname{dim} X$ satisfies $Y \cong \mathbb{P}^{1} \times \cdots \times \mathbb{P}^{1}$. Then, either $X \cong \mathbb{P}^{1} \times \cdots \times \mathbb{P}^{1}$, or $X$ is a double cover of $\mathbb{P}^{1} \times \cdots \times \mathbb{P}^{1}$ branched along a reduced divisor of type $(2, \ldots, 2)$.

In order to prove Theorem 1.3, we are led to study finite morphisms between some Del Pezzo surfaces (see Propositions 7.2 and 8.3). Beauville classified in [4] the smooth Del Pezzo surfaces which admit an endomorphism of degree $>1$.

Proposition 1.5 ([4, Proposition 3]). A smooth Del Pezzo surface $S$ admits an endomorphism of degree $>1$ if and only if $K_{S}^{2} \geqslant 6$.

In section 10, we address finite morphisms between smooth Del Pezzo surfaces. Our result is the following. We believe that this result is interesting on its own and that it will be useful when considering Fano manifolds with $\operatorname{Nef}(X)=\operatorname{Psef}(X)$ and arbitrary Picard number.

Theorem 1.6. Let $S$ and $T$ be smooth Del Pezzo surfaces with $K_{S}^{2}<K_{T}^{2}$, and let $f: S \rightarrow T$ be a finite morphism. Then $K_{T}^{2} \geqslant 8$.

\section{Notation and conventions}

Throughout this paper we work over the field of complex numbers. Varieties are always assumed to be reduced and irreducible.

We denote by $\operatorname{Sing}(X)$ the singular locus of a variety $X$. 
Let $X$ be a normal projective variety, and $B=\sum a_{i} B_{i}$ an effective $\mathbb{Q}$ divisor on $X$, i.e., $B$ is a nonnegative $\mathbb{Q}$-linear combination of distinct prime Weil divisors $B_{i}$ 's on $X$. Suppose that $K_{X}+B$ is $\mathbb{Q}$-Cartier, i.e., some nonzero multiple of it is a Cartier divisor. Let $\mu: \tilde{X} \rightarrow X$ be a log resolution of the pair $(X, B)$. This means that $\tilde{X}$ is a smooth projective variety, $\mu$ is a birational projective morphism whose exceptional locus is the union of prime divisors $E_{i}$ 's, and the divisor $\sum E_{i}+\tilde{B}$ has simple normal crossing support, where $\tilde{B}$ denotes the strict transform of $B$ in $X$. There are uniquely defined rational numbers $a\left(E_{i}, X, B\right)$ 's such that

$$
K_{\tilde{X}}+\tilde{B}=\mu^{*}\left(K_{X}+B\right)+\sum a\left(E_{i}, X, B\right) E_{i} .
$$

The $a\left(E_{i}, X, B\right)$ 's do not depend on the $\log$ resolution $\mu$, but only on the valuations associated to the $E_{i}$ 's. We say that $(X, B)$ is canonical (respectively, log terminal or $k l t$ ) if all $a_{i} \leqslant 1$ (respectively, $a_{i}<1$ ), and, for some log resolution $\mu: \tilde{X} \rightarrow X$ of $(X, B), a\left(E_{i}, X, B\right) \geqslant 0$ (respectively, $a\left(E_{i}, X, B\right) \geqslant$ $-1)$ for every $\mu$-exceptional prime divisor $E_{i}$. We say that $(X, B)$ is $\log$ canonical if all $a_{i} \leqslant 1$, and, for some $\log$ resolution $\mu: \tilde{X} \rightarrow X$ of $(X, B)$, $a\left(E_{i}, X, B\right) \geqslant-1$ for every $\mu$-exceptional prime divisor $E_{i}$. If these conditions hold for some $\log$ resolution of $(X, B)$, then they hold for every log resolution of $(X, B)$. We say that $X$ is canonical (respectively log canonical) if so is $(X, 0)$. We say that $X$ is Gorenstein, if $X$ is locally Cohen-Macaulay and $K_{X}$ is Cartier. Note that if $X$ is Gorenstein, then $X$ is canonical if and only if $X$ is klt. We say that $X$ is $\mathbb{Q}$-Gorenstein, if $K_{X}$ is $\mathbb{Q}$-Cartier.

Let $X$ be a normal projective variety such that $K_{X}$ is $\mathbb{Q}$-Cartier. We say that $X$ is $\mathbb{Q}$-Fano if $-K_{X}$ is ample. We say that a normal surface $S$ is a Del Pezzo surface if $S$ is $\mathbb{Q}$-Fano with canonical singularitites. Note that a normal surface $S$ is canonical if and only if $S$ has Du Val singularities (see [19, Theorem 4.5]).

Let $X$ be a normal projective variety, and $B$ an effective $\mathbb{Q}$-divisor on $X$ such that $K_{X}+B$ is $\mathbb{Q}$-Cartier. We say that $(X, B)$ is $\mathbb{Q}$-Fano if $-\left(K_{X}+B\right)$ is ample.

If $\mathscr{E}$ is a locally free sheaf of $\mathscr{O}_{X}$-modules on a variety $X$, we denote by $\mathbb{P}_{X}(\mathscr{E})$ the Grothendieck projectivization $\operatorname{Proj}_{X}(\operatorname{Sym}(\mathscr{E}))$, and by $\mathscr{O}_{\mathbb{P}_{X}(\mathscr{E})}(1)$ its tautological line bundle.

Given a positive integer $m$, we denote by $\mathbb{F}_{m}$ the surface $\mathbb{P}_{\mathbb{P}^{1}}\left(\mathscr{O}_{\mathbb{P}^{1}} \oplus\right.$ $\left.\mathscr{O}_{\mathbb{P}^{1}}(-m)\right)$.

Given line bundles $\mathscr{L}_{1}$ and $\mathscr{L}_{2}$ on varieties $X_{1}$ and $X_{2}$, we denote by $\mathscr{L}_{1} \otimes \mathscr{L}_{2}$ the line bundle $\pi_{1}^{*} \mathscr{L}_{1} \otimes \pi_{2}^{*} \mathscr{L}_{2}$ on $X_{1} \times X_{2}$, where $\pi_{1}$ and $\pi_{2}$ are the projections onto $X_{1}$ and $X_{2}$, respectively. 


\section{Double covers}

In this section we gather some properties of double covers of smooth (projective) varieties.

3.1. Let $f: X \rightarrow Y$ be a finite surjective morphism of degree 2. Suppose that $X$ is Cohen-Macaulay and $Y$ is smooth. Then there exist a line bundle $\mathscr{L}$ on $Y$, a section $s \in H^{0}\left(Y, \mathscr{L}^{\otimes 2}\right)$, and an isomorphism $f_{*} \mathscr{O}_{X} \cong \mathscr{O}_{Y} \oplus$ $\mathscr{L}^{\otimes-1}$ of $\mathscr{O}_{Y}$-algebras, where the structure of $\mathscr{O}_{Y}$-algebra on $\mathscr{O}_{Y} \oplus \mathscr{L}^{\otimes-1}$ is induced by $s^{\vee}: \mathscr{L}^{\otimes-2} \rightarrow \mathscr{O}_{Y}$. We refer to [8] for details. This implies that $X$ is Gorenstein with dualizing sheaf $\omega_{X} \cong f^{*}\left(\omega_{Y} \otimes \mathscr{L}\right)$. If moreover $Y$ is projective, then $X$ is $\mathbb{Q}$-Fano if and only if $\left(\omega_{Y} \otimes \mathscr{L}\right)^{\otimes-1}$ is an ample line bundle.

Denote by $B$ the divisor of zeroes of $s$. A straightforward local computation shows that $X$ is normal if and only if $B$ is reduced. By [19, Proposition 5.20], $X$ is canonical if and only if $\left(Y, \frac{1}{2} B\right)$ is klt.

Lemma 3.2. Fix an integer $n \geqslant 1$, and let $f: X \rightarrow\left(\mathbb{P}^{1}\right)^{n}$ be a double cover branched along a reduced divisor $B$. Then $X$ is $\mathbb{Q}$-Fano if and only if $B$ has type $\left(2 d_{1}, \ldots, 2 d_{n}\right)$ with $d_{i} \in\{0,1\}$ for each $i \in\{1, \ldots, n\}$.

Remark 3.3. In the setup of Lemma 3.2, denote by $m$ the cardinality of the set $\left\{1 \leqslant i \leqslant n \mid d_{i}=0\right\}$. Then $X \cong\left(\mathbb{P}^{1}\right)^{m} \times Y$ where $Y$ is a double cover of $\left(\mathbb{P}^{1}\right)^{n-m}$ branched along a reduced divisor of type $(2, \ldots, 2)$.

Lemma 3.4. Let $X$ and $Y$ be projective Fano manifolds, and let $X \rightarrow Y$ be a double cover branched along an ample divisor. Suppose furthermore that $\operatorname{dim} X=\operatorname{dim} Y \geqslant 3$. Then $\rho(X)=\rho(Y)$.

Proof. From 3.1 and [20, Theorem 2.1], we conclude that $b_{2}(X)=b_{2}(Y)$. Since $X$ and $Y$ are Fano manifolds, we also have $\rho(X)=b_{2}(X)$ and $\rho(Y)=$ $b_{2}(Y)$, proving the lemma.

Corollary 3.5. Let $X$ be a smooth double cover of $\mathbb{P}^{1} \times \cdots \times \mathbb{P}^{1}$ branched along a reduced divisor $B$ of type $(2, \ldots, 2)$ with $\operatorname{dim} X \geqslant 3$. Then $\rho(X)=$ $\operatorname{dim} X$.

Proof. This follows from Lemma 3.4 together with Lemma 3.2.

The following example shows that the statement of Lemma 3.4 does not hold for surfaces. 
Example 3.6. Let $S$ be a double cover of $\mathbb{P}^{1} \times \mathbb{P}^{1}$ branched along a smooth divisor of type $(2,2)$. Then $S$ is a (smooth) Del Pezzo surface of degree $K_{S}^{2}=4$, and $\rho(S)=6$.

We will need the following observations.

Lemma 3.7. Let $(R, \mathfrak{m})$ be a regular local ring, and let $f_{1}, f_{2} \in \mathfrak{m}$ be coprime elements. Then the hypersurface ring

$$
R[t]_{(t)} /\left(t^{2}-f_{1} f_{2}\right)
$$

is not factorial.

Proof. Let $\mathfrak{n}$ be the maximal ideal of the ring $\mathscr{O}=R[t]_{(t)} /\left(t^{2}-f_{1} f_{2}\right)$. Note that $t$ is irreducible since $t \in \mathfrak{n} \backslash \mathfrak{n}^{2}$. To prove the lemma, suppose to the contrary that $\mathscr{O}$ is factorial. Since $t^{2}=f_{1} f_{2}$ and $f_{1}$ and $f_{2}$ are coprime, we conclude that $f_{1}$ or $f_{2}$ is a unit. This yields a contradiction.

Lemma 3.8. Let $a, b, c, d, e$, and $f$ be complex numbers. The hypersurface ring

$$
\mathbb{C}[x, y, t]_{(x, y, t)} /\left(t^{2}-a x^{2}-b x y-c y^{2}-d x^{2} y-e x y^{2}-f x^{2} y^{2}\right)
$$

is not factorial.

Proof. Let $a_{1} \in \mathbb{C}$ such that $a=a_{1}^{2}$. The hypersurface $X \subset \mathbb{A}^{3}$ given by equation

$$
t^{2}-a x^{2}-b x y-c y^{2}-d x^{2} y-e x y^{2}-f x^{2} y^{2}=0
$$

contains $(0,0,0)$ in its singular locus. Moreover, the line given by equations

$$
y=t-a_{1} x=0
$$

is a smooth hypersurface on $X$ passing through $(0,0,0)$. Therefore $X$ is not locally factorial at $(0,0,0)$.

The next result is an immediate consequence of Lemma 3.8.

Corollary 3.9. Let $X$ be a double cover of $\mathbb{P}^{1} \times \mathbb{P}^{1}$ branched along a reduced divisor of type $(2,2)$. Then $X$ is locally factorial if and only if it is smooth. 
Lemma 3.10. Let $f: X \rightarrow Y$ be a finite surjective morphism of degree 2 with $X$ Cohen-Macaulay and $Y$ smooth. If $\operatorname{dim}(\operatorname{Sing}(X)) \leqslant \operatorname{dim} X-4$, then $X$ is locally factorial.

Proof. The lemma follows from 3.1 and [15, Exposé XI Corollaire 3.14].

The following example shows that the statement of Lemma 3.10 becomes wrong if one relaxes the assumption on $\operatorname{dim}(\operatorname{Sing}(X))$.

Example 3.11. Let $B \subset \mathbb{P}^{1} \times \mathbb{P}^{1} \times \mathbb{P}^{1}$ be defined by equation

$$
x_{0}^{2} y_{0}^{2} z_{1}^{2}+z_{0}^{2} x_{1}^{2} y_{1}^{2}+z_{0} z_{1}\left(x_{0} x_{1} y_{1}^{2}+y_{0} y_{1} x_{1}^{2}\right)=0
$$

and let $X$ be the double cover of $\mathbb{P}^{1} \times \mathbb{P}^{1} \times \mathbb{P}^{1}$ branched along $B$. Denote by $f: X \rightarrow \mathbb{P}^{1} \times \mathbb{P}^{1} \times \mathbb{P}^{1}$ the natural morphism. Set $P=(0,1) \times(0,1) \times$ $(0,1) \in \mathbb{P}^{1} \times \mathbb{P}^{1} \times \mathbb{P}^{1}$ and $Q=f^{-1}(P) \in X$. A straightforward computation shows that $X$ is normal and singular at $Q$. The surface $S=f^{-1}\left(\left\{z_{0}=0\right\}\right)$ is the double cover of $\mathbb{P}^{1} \times \mathbb{P}^{1}$ branched along the divisor defined by equation $x_{0}^{2} y_{0}^{2}=0$. Hence, $S$ is the union of two copies of $\left\{z_{0}=0\right\} \cong \mathbb{P}^{1} \times \mathbb{P}^{1}$ passing through $Q$. Therefore $X$ is not locally factorial at $Q$.

Lemma 3.12. Let $X$ be a double cover of $\mathbb{P}^{1} \times \cdots \times \mathbb{P}^{1}$ branched along a reduced divisor $B$ of type $(2, \ldots, 2)$. If $\operatorname{dim} \operatorname{Sing}(X) \leqslant \operatorname{dim} X-3$, then $\pi_{1}(X \backslash \operatorname{Sing}(X)) \cong\{1\}$.

Proof. Set $n=\operatorname{dim} X$. By Lemma 3.2, $X$ is $\mathbb{Q}$-Fano. If $n=1$, then $X \cong \mathbb{P}^{1}$, and hence $\pi_{1}(X)=\{1\}$ as claimed. Suppose that $n \geqslant 2$. Then apply Lemma 3.13 below to any projection $f_{1}: X \rightarrow\left(\mathbb{P}^{1}\right)^{n-1}$ such that $f_{1}(\operatorname{Supp}(B))=$ $\left(\mathbb{P}^{1}\right)^{n-1}$.

Lemma 3.13. Let $Y$ be a $\mathbb{Q}$-Fano variety of dimension $n \geqslant 2$, and let $\pi: Y \rightarrow T$ be a surjective equidimensional morphism with connected fibers onto a normal variety such that $\operatorname{dim} \pi(\operatorname{Sing}(Y)) \leqslant \operatorname{dim} T-2$. Then $\pi_{1}(T \backslash$ $\operatorname{Sing}(T)) \cong \pi_{1}(Y \backslash \operatorname{Sing}(Y))$.

Proof. Let $F$ be a general fiber of $\pi$. Then $F$ is a Fano manifold, and therefore $F$ is rationally chain connected (see [6] and [18]). Consider a smooth general complete intersection curve $B \subset T$, and set $Z=\pi^{-1}(B)$. Then $Z$ is smooth, and the morphism $\pi_{Z}: Z \rightarrow B$ induced by the restriction of $\pi$ to $Z$ has rationally chain connected general fibers. By [14], we conclude that the scheme theoretic fiber $\pi_{Z}^{-1}(b)$ has a smooth point for each $b \in B$. Therefore, there exists a codimension $\geqslant 2$ closed subset $G$ of $T$ such that 
- $\operatorname{Sing}(T) \cup \pi(\operatorname{Sing}(Y)) \subset G$,

- for each $t \in T \backslash G$, the scheme theoretic fiber $\pi^{-1}(p)$ has a smooth point.

Thus, by [23, Lemma 1.5], there is an exact sequence

$$
\pi_{1}(F) \rightarrow \pi_{1}\left(Y \backslash \pi^{-1}(G)\right) \rightarrow \pi_{1}(T \backslash G) \rightarrow 1 .
$$

Now, we have $\pi_{1}(T \backslash G) \cong \pi_{1}(T \backslash \operatorname{Sing}(T))$ and $\pi_{1}\left(Y \backslash \pi^{-1}(G)\right) \cong \pi_{1}(Y \backslash$ Sing $(Y))$ since $S$ and $\pi^{-1}(G)$ have codimension $\geqslant 2$ respectively. Fano manifolds being simply connected, we conclude that $\pi_{1}(T \backslash \operatorname{Sing}(T)) \cong \pi_{1}(Y \backslash$ $\operatorname{Sing}(Y))$, proving the lemma.

Remark 3.14. Example 6.2 shows that the statements of Lemmata 3.12 and 3.13 becomes wrong if one relaxes the assumption on $\operatorname{dim} \pi(\operatorname{Sing}(Y))$.

\section{Mori point of view}

Let $\varphi: X \rightarrow Y$ be an arbitrary surjective morphism of normal projective varieties. The natural morphism $\varphi^{*}: \mathrm{N}^{1}(Y) \rightarrow \mathrm{N}^{1}(X)$ is injective,

$$
\begin{aligned}
\varphi^{*}(\operatorname{Nef}(Y)) & =\operatorname{Nef}(X) \cap \varphi^{*}\left(\mathrm{~N}^{1}(Y)\right), \quad \text { and } \\
\varphi^{*}(\operatorname{Psef}(Y)) & =\operatorname{Psef}(X) \cap \varphi^{*}\left(\mathrm{~N}^{1}(Y)\right) .
\end{aligned}
$$

Let now $X$ and $Y$ be normal projective varieties. If $\operatorname{Nef}(X \times Y)=$ $\operatorname{Psef}(X \times Y)$, then it is easy to see that $\operatorname{Nef}(X)=\operatorname{Psef}(X)$ and $\operatorname{Nef}(Y)=$ $\operatorname{Psef}(Y)$. We have the following partial converse to the above statement.

Lemma 4.1. Let $X$ and $Y$ be normal projective varieties. Suppose that $h^{1}\left(X, \mathscr{O}_{X}\right)=0$. If $\operatorname{Nef}(X)=\operatorname{Psef}(X)$ and $\operatorname{Nef}(Y)=\operatorname{Psef}(Y)$, then $\operatorname{Nef}(X \times$ $Y)=\operatorname{Psef}(X \times Y)$.

Proof. Since $h^{1}\left(X, \mathscr{O}_{X}\right)=0$, we have $\operatorname{Pic}(X \times Y) \cong \operatorname{Pic}(X) \times \operatorname{Pic}(Y)$. It is easy to see that $\operatorname{Nef}(X \times Y) \cong \operatorname{Nef}(X) \times \operatorname{Nef}(Y)$, and $\operatorname{Psef}(X \times Y) \cong$ $\operatorname{Psef}(X) \times \operatorname{Psef}(Y)$. The lemma follows.

We will make use of the following elementary lemma.

Lemma 4.2. Let $f: X \rightarrow Y$ be an arbitrary surjective morphism of normal projective varieties. If $\operatorname{Nef}(X)=\operatorname{Psef}(X)$, then $\operatorname{Nef}(Y)=\operatorname{Psef}(Y)$.

Proof. The lemma follows from the projection formula. 
Remark 4.3. Let $\varphi: X \rightarrow \mathbb{P}^{1} \times \mathbb{P}^{1} \times \mathbb{P}^{1}$ be a double cover branched along a smooth divisor of type $(2,2,2)$, and let $\pi: X \rightarrow \mathbb{P}^{1}$ be a projection. Then a general fiber $F$ of $\pi$ is a smooth Del Pezzo surface of degree 4 with $\operatorname{Nef}(F) \subsetneq$ $\operatorname{Psef}(F)$.

The following observation will prove to be crucial.

Lemma 4.4. Let $(X, B)$ be a $\mathbb{Q}$-Fano pair with $\mathbb{Q}$-factorial log canonical singularities. Then the following conditions are equivalent.

1) $\operatorname{Nef}(X)=\operatorname{Psef}(X)$.

2) Any effective Cartier divisor on $X$ is semiample.

3) Any elementary contraction $X \rightarrow Y$ satisfies $\operatorname{dim} Y<\operatorname{dim} X$.

4) Any non-trivial contraction $X \rightarrow Y$ satisfies $\operatorname{dim} Y<\operatorname{dim} X$.

Proof. (1) $\Rightarrow(2)$ Let $E$ be an effective Cartier divisor on $X$. Then $E$ is nef and thus $E-\left(K_{X}+B\right)$ is ample. By [10, Theorem 13.1], we conclude that $E$ is semiample.

(2) $\Rightarrow$ (3) Let $\varphi: X \rightarrow Y$ be an elementary contraction. We argue by contradiction, and assume that $\operatorname{dim} Y=\operatorname{dim} X$. Set $C=\varphi_{*} B$. Let $A$ be an ample Cartier divisor on $Y$ such that $K_{Y}+C+A$ is $\mathbb{Q}$-ample, and let $m$ be a positive integer such that $m B \in Z^{1}(X)_{\mathbb{Z}}$ and $h^{0}\left(Y, \mathscr{O}_{Y}\left(m\left(K_{Y}+C+\right.\right.\right.$ A)) $\geqslant 1$.

Suppose first that $\operatorname{dim} \operatorname{Exc}(\varphi)=\operatorname{dim} X-1$. Then $F=\operatorname{Exc}(\varphi)$ is irreducible, $Y$ is $\mathbb{Q}$-factorial, and $K_{X}+B=\varphi^{*}\left(K_{Y}+C\right)+a F$ for some rational number $a \geqslant 0$. Assume in addition that $m a \in \mathbb{Z}$. Then

$$
\begin{aligned}
& h^{0}\left(X, \mathscr{O}_{X}\left(m\left(K_{X}+B+\varphi^{*} A\right)\right)\right) \\
= & h^{0}\left(X, \varphi^{*} \mathscr{O}_{Y}\left(m\left(K_{Y}+C+A\right)+m a F\right)\right) \\
= & h^{0}\left(Y, \mathscr{O}_{Y}\left(m\left(K_{Y}+C+A\right)\right)\right) \quad \text { since } m a \in \mathbb{N} \\
\geqslant & 1 .
\end{aligned}
$$

Thus, there exists an effective Cartier divisor $E \sim_{\mathbb{Q}} K_{X}+B+\varphi^{*} A$. This yields a contradiction since $K_{X}+B+\varphi^{*} A$ is not nef. 
Suppose now that $\operatorname{dim} \operatorname{Exc}(\varphi) \leqslant \operatorname{dim} X-2$. Set $X^{\circ}=X \backslash \operatorname{Exc}(\varphi)$ and $Y^{\circ}=Y \backslash \varphi(\operatorname{Exc}(\varphi))$. Then

$$
\begin{array}{rlrl} 
& h^{0}\left(X, \mathscr{O}_{X}\left(m\left(K_{X}+B+\varphi^{*} A\right)\right)\right) & \\
= & h^{0}\left(X^{\circ}, \mathscr{O}_{X}\left(m\left(K_{X}+B+\varphi^{*} A\right)\right)\right) & & \text { since } \operatorname{codim} \operatorname{Exc}(\varphi) \geqslant 2 \\
= & h^{0}\left(Y^{\circ}, \mathscr{O}_{Y}\left(m\left(K_{Y}+C+A\right)\right)\right) & & \text { since } X^{\circ} \cong Y^{\circ} \\
= & h^{0}\left(Y, \mathscr{O}_{Y}\left(m\left(K_{Y}+C+A\right)\right)\right) & & \text { since } \operatorname{codim} \varphi(\operatorname{Exc}(\varphi)) \geqslant 2 \\
\geqslant & 1 . &
\end{array}
$$

We conclude as before that there exists an effective divisor $E \sim_{\mathbb{Q}} K_{X}+B+$ $\varphi^{*} A$, yielding a contradiction since $K_{X}+B+\varphi^{*} A$ is not nef. This proves that $\operatorname{dim} Y<\operatorname{dim} X$.

$(3) \Rightarrow(4)$ is obvious.

$(4) \Rightarrow(1)$ Let $E$ be an effective Cartier divisor. Suppose that $E$ is not nef. Then there exists an extremal ray $R \subset \mathrm{NE}(X)$ such that $E \cdot C<0$ for every curve $C$ with $[C] \in R$. Let $\varphi: X \rightarrow Y$ be the corresponding contraction. Then we must have $\operatorname{Exc}(\varphi) \subset \operatorname{Supp}(E)$, yielding a contradiction, and completing the proof of the lemma.

To prove Theorem 1.1, we will argue by induction on $\operatorname{dim} X$. We will make use of the following result.

Lemma 4.5. Let $(X, B)$ be a $\mathbb{Q}$-Fano pair with log terminal (respectively, $\log$ canonical) singularities. Let $\varphi: X \rightarrow Y$ be any contraction. Then there exists an effective $\mathbb{Q}$-divisor $B_{Y}$ on $Y$ such that $\left(Y, B_{Y}\right)$ is $\mathbb{Q}$-Fano with log terminal (respectively, log canonical) singularities.

Proof. Let $A$ be an ample $\mathbb{Q}$-divisor on $Y, 0<\varepsilon \ll 1$ a rational number, and $C \sim \mathbb{Q}-\left(K_{X}+B\right)-\varepsilon \varphi^{*} A$ an effective $\mathbb{Q}$-divisor such that $(X, B+C)$ is klt. Suppose that $(X, B)$ has $\log$ terminal singularities. By [1, Theorem 4.1] applied to $(X, B+C)$, there exists an effective $\mathbb{Q}$-divisor $B_{Y}$ on $Y$ such that $\left(Y, B_{Y}\right)$ is klt and $K_{Y}+B_{Y} \sim_{\mathbb{Q}}-\varepsilon A$. If $(X, B)$ has $\log$ canonical singularities, then one only needs to replace the use of $[1$, Theorem 4.1] with [11, Therorem 3.4].

The same argument used in the proof of [17, Lemma 5.1.5] shows that the following lemma holds. One only needs to replace the use of [17, Lemma 3.2.5] with [10, Theorem 1.1]. 
Lemma 4.6. Let $(X, B)$ be a pair with log canonical singularities, and let $\varphi: X \rightarrow Y$ be an elementary Mori contraction with $\operatorname{dim} Y<\operatorname{dim} X$. If $X$ is locally factorial (respectively, $\mathbb{Q}$-factorial), then $Y$ is locally factorial (respectively, $\mathbb{Q}$-factorial).

Remark 4.7. Let $(X, B)$ be a pair with log canonical singularities. Then the conclusion of Lemma 4.6 holds for any quasi-elementary Mori contraction $\varphi: X \rightarrow Y$ with $\operatorname{dim} Y<\operatorname{dim} X$. We refer to [7] for the definition of quasielementary Mori contractions.

Corollary 4.8. Let $(X, B)$ be a $\mathbb{Q}$-Fano pair with log canonical singularities satisfying $\operatorname{Nef}(X)=\operatorname{Psef}(X)$. Let $V$ be a face of $\mathrm{NE}(X)$, and denote by $\varphi: X \rightarrow Y$ the corresponding contraction. If $X$ is locally factorial, then so is $Y$.

Proof. We argue by induction on $\rho(X / Y)=\rho(X)-\rho(Y) \geqslant 0$. If $\rho(X / Y)=$ 0 , then $X \cong Y$ and there is nothing to prove. Suppose that $\rho(X / Y) \geqslant 1$. Let $R \subset V$ be an extremal ray of $\mathrm{NE}(X)$, and denote by $\psi: X \rightarrow Z$ the corresponding contraction. There exists a morphism $\xi: Z \rightarrow Y$ such that $\varphi=$ $\xi \circ \psi$. From Lemma 4.2, we conclude that $\operatorname{Nef}(Z)=\operatorname{Psef}(Z)$. By Lemma 4.5, there exists an effective $\mathbb{Q}$-divisor $B_{Z}$ on $Z$ such that $\left(Z, B_{Z}\right)$ is $\mathbb{Q}$-Fano with $\log$ canonical singularities. By Lemma 4.4, we have $\operatorname{dim} Z<\operatorname{dim} Y$, and Lemma 4.6 tells us that $Z$ is locally factorial. Notice that $\rho(Z / Y)=$ $\rho(X / Y)-1<\rho(X / Y)$. The lemma follows.

It follows from [27, Theorem 2.2] that a Fano manifold with $\operatorname{Nef}(X)=$ $\operatorname{Psef}(X)$ satisfies $\rho(X) \leqslant \operatorname{dim} X$. We now extend this result to mildly singular varieties.

Lemma 4.9. Let $(X, B)$ be a $\mathbb{Q}$-Fano pair with log canonical singularities satisfying $\operatorname{Nef}(X)=\operatorname{Psef}(X)$.

1) Then $\rho(X) \leqslant \operatorname{dim} X$.

2) Suppose moreover that $\rho(X)=\operatorname{dim} X$. Then $\mathrm{NE}(X)$ is simplicial, and the following holds. Let $V$ be a face of $\mathrm{NE}(X)$, and denote by $\varphi: X \rightarrow$ $Y$ the corresponding contraction. Then $\varphi$ is equidimensional and $\operatorname{dim} Y=\operatorname{dim} X-\operatorname{dim} V=\rho(Y)$.

Proof. Set $n=\operatorname{dim} X$. Suppose that $\rho(X)=\operatorname{dim} \mathrm{NE}(X) \geqslant n$. Let $R_{1}, \ldots$, $R_{n-1}$ be extremal rays of $\mathrm{NE}(X)$ such that $V_{i}=R_{1}+\cdots+R_{i}$ is a face of $\mathrm{NE}(X)$ with $\operatorname{dim}\left(R_{1}+R_{2}+\cdots+R_{i}\right)=i$ for each $1 \leqslant i \leqslant n-1$. Denote by 
$\varphi_{i}: X \rightarrow Y_{i}$ the contraction of $V_{i}$. We have $\operatorname{dim} Y_{i} \leqslant n-i$ by Lemma 4.4(4). Let $i_{0}$ be the smallest number $1 \leqslant i \leqslant n-1$ such that $\operatorname{dim} Y_{i_{0}} \leqslant 1$. By $[10$, Theorem 16.4(3)], we have $\rho\left(Y_{i}\right)=\rho(X)-i$, and therefore

$$
\rho(X)-n+1 \leqslant \rho(X)-i_{0}=\rho\left(Y_{i_{0}}\right) \leqslant 1 .
$$

We conclude that $\rho(X)=n$ and $i_{0}=n-1$, proving (1).

We proceed to prove (2). Suppose that $\rho(X)=n$.

We first show that $\operatorname{NE}(X)$ is simplicial. Suppose otherwise and consider a 2-dimensional face $W$ of $\operatorname{NE}(X)$ such that $V_{n-1} \cap W=\{0\}$. Denote by $\psi: X \rightarrow T$ the corresponding contraction. Let $F$ and $G$ be fibers of $\varphi_{n-1}$ and $\psi$ respectively. Since $\operatorname{dim} Y_{n-1}=1$ and $\operatorname{dim} T \leqslant n-2$, we must have $\operatorname{dim}(F \cap G) \geqslant 1$, yielding a contradiction. This proves that $\mathrm{NE}(X)$ is simplicial.

Let $V$ be a face of $\mathrm{NE}(X)$, and denote by $\varphi: X \rightarrow Y$ the corresponding contraction. We have $\rho(Y)=\rho(X)-\operatorname{dim} V=n-\operatorname{dim} V$. Thus there exists a face $W$ of $\operatorname{NE}(X)$ with $\operatorname{dim} W=\rho(Y)$ and $V \cap W=\{0\}$. Denote by $\psi: X \rightarrow T$ the corresponding contraction. By Lemma 4.4(4), we must have

$$
\operatorname{dim} Y \leqslant n-\operatorname{dim} V=\rho(Y)
$$

and

$$
\operatorname{dim} T \leqslant n-\operatorname{dim} W=n-\rho(Y) .
$$

Since $V \cap W=\{0\}$, we conclude that $\varphi$ and $\psi$ are equidimensional, $\operatorname{dim} Y=$ $n-\operatorname{dim} V$, and $\operatorname{dim} T=n-\operatorname{dim} W$. This completes the proof of the lemma.

Remark 4.10. In the setup of the proof of Lemma 4.9, suppose moreover that $X$ is smooth. Then $Y_{i}$ is smooth and $\varphi_{i}$ is a conic bundle for each $1 \leqslant i \leqslant n$ by [2, Theorem 3.1].

Remark 4.11. One might ask whether Lemma 4.9 holds for a larger class of singular varieties. What we actually proved is the following. Let $X$ be a normal projective variety with rational polyhedral Mori cone $\mathrm{NE}(X)$. Suppose that every face $V$ of $\mathrm{NE}(X)$ corresponds to a surjective morphism with connected fibers $\varphi: X \rightarrow Y$ onto a normal projective variety such that $\varphi$ contracts precisely those curves on $X$ with class in $V$. Suppose furthermore that $\rho(Y)=\rho(X)-\operatorname{dim} V$ and $\operatorname{dim} Y<\operatorname{dim} X$ for each face $V$ of $\operatorname{NE}(X)$. If $\operatorname{Nef}(X)=\operatorname{Psef}(X)$, then $X$ satisfies the conclusions of Lemma 4.9. 
The same argument used in the proof of Lemma 4.9 above shows that the following lemmata hold.

Lemma 4.12. With the assumptions as in Lemma 4.9, suppose furthermore that there is a contraction $\varphi: X \rightarrow Y$ such that $\operatorname{dim} Y=\rho(Y)$. Then, there exists a contraction $\psi: X \rightarrow T$ such that the induced morphism $(\varphi, \psi)$ : $X \rightarrow Y \times T$ is finite and surjective. In particular, $\varphi$ and $\psi$ are equidimensional.

Lemma 4.13. With the assumptions as in Lemma 4.9, suppose furthermore that there is an elementary contraction $\varphi: X \rightarrow Y$ with $\operatorname{dim} Y=$ $\rho(X)-1=\rho(Y)$. Denote by $R$ the corresponding extremal ray. Then $\mathrm{NE}(X)$ is simplicial, and the following holds. Let $W$ be a face of $\mathrm{NE}(X)$, and denote by $\psi: X \rightarrow T$ the corresponding contraction. Then $\psi$ is equidimensional, and either $\operatorname{dim} T=\operatorname{dim} X-\operatorname{dim} W$ if $R \not \subset W$ or $\operatorname{dim} T=\rho(X)-\operatorname{dim} W=\rho(T)$ if $R \subset W$.

\section{Fibrations on $\mathbb{Q}$-Fano threefolds with canonical Gorenstein singularities}

In this section we provide a technical tool for the proof of the main results.

5.1. Let $N \geqslant 2$ be an integer. We say that $Y \subset \mathbb{P}^{N}$ is a variety of minimal degree if $Y$ is nondegenerate and $\operatorname{deg}(Y)=$ codim $Y+1$. Surfaces of minimal degree were classified by Del Pezzo. Bertini then obtained a similar classification for varieties of any dimension. If $Y \subset \mathbb{P}^{N}$ is a variety of minimal degree and $\operatorname{codim} Y \geqslant 2$, then $Y$ is either a rational normal scroll or a cone over the Veronese surface in $\mathbb{P}^{2} \subset \mathbb{P}^{5}$. A rational normal scroll is a cone over a smooth linearly normal variety fibered over $\mathbb{P}^{1}$ by linear spaces. Note that the Veronese surface contains no lines, and thus a cone over the Veronese surface cannot contain a linear space of codimension 1. A rational normal scroll contains a pencil of linear spaces of codimension 1 . This pencil is unique if and only if $Y$ is not a cone over $\mathbb{P}^{1} \times \mathbb{P}^{1} \subset \mathbb{P}^{3}$.

5.2. Let $X$ be a 3 -dimensional $\mathbb{Q}$-Fano variety with Gorenstein canonical singularities. Then we have

$$
h^{0}\left(X, \mathscr{O}_{X}\left(-K_{X}\right)\right)=-\frac{1}{2} K_{X}^{3}+3 .
$$


Set $N=-\frac{1}{2} K_{X}^{3}+2$, and notice that $N \geqslant 3$. Denote by $\varphi: X \rightarrow \rightarrow \mathbb{P}^{N}$ the rational map given by the complete linear system $\left|-K_{X}\right|$, and set $Y=$ $\varphi(X)$.

The classification of 3 -dimensional $\mathbb{Q}$-Fano varieties $X$ with Gorenstein canonical singularities satisfying $\operatorname{Bs}\left(-K_{X}\right) \neq \emptyset$ was established in [16]. Those with $\operatorname{Bs}\left(-K_{X}\right)=\emptyset$ and $-K_{X}$ not very ample were classified in [25, Theorem 1.5]. We do not include the classification here. Instead, we state the properties that we need. If $\operatorname{Bs}\left(-K_{X}\right) \neq \emptyset$, then $Y \subset \mathbb{P}^{N}$ is a variety of minimal degree with $\operatorname{dim} Y=2$, and the rational map $\varphi: X \rightarrow Y$ has connected fibers. If $\operatorname{Bs}\left(-K_{X}\right)=\emptyset$ and $-K_{X}$ is not very ample, then $Y \subset \mathbb{P}^{N}$ is a variety of minimal degree, and the finite morphism $\varphi: X \rightarrow Y$ has degree 2 .

We are now ready to state and prove the main result of this section.

Lemma 5.3. Let $X$ be a 3 -dimensional $\mathbb{Q}$-Fano variety with Gorenstein canonical singularities. Then there is at most one fibration $f: X \rightarrow \mathbb{P}^{1}$ with general fiber $S$ satisfying $K_{S}^{2} \leqslant 2$.

Proof. Denote by $\varphi: X \rightarrow \mathbb{P}^{N}$ the rational map given by the complete linear system $\left|-K_{X}\right|$, and set $Y=\varphi(X) \subset \mathbb{P}^{N}$.

Let $f: X \rightarrow \mathbb{P}^{1}$ be a surjective morphism with connected fibers, and general fiber $S$. Suppose that $K_{S}^{2} \leqslant 2$. We will show that there exists a rational fibration $g: Y \rightarrow \mathbb{P}^{1}$ by linear spaces such that $g \circ \varphi$ factors through $f$. Notice that $S$ has canonical singularities. Denote by $\psi: S \rightarrow \mathbb{P}^{M}$ the rational map given by the complete linear system $\left|-K_{S}\right|$, where $M+1=$ $h^{0}\left(S, \mathscr{O}_{S}\left(-K_{S}\right)\right)=K_{S}^{2}+1$. Then $\operatorname{Bs}\left(-K_{S}\right) \neq \emptyset$ if and only if $K_{S}^{2}=1$. If $K_{S}^{2}=2$, then $\psi: S \rightarrow \mathbb{P}^{2}$ is a double cover. We conclude that $-K_{S}$ is not very ample, and therefore neither is $-K_{X}$.

The restriction map $r: H^{0}\left(X, \mathscr{O}_{X}\left(-K_{X}\right)\right) \rightarrow H^{0}\left(S, \mathscr{O}_{S}\left(-K_{S}\right)\right)$ induces a commutative diagram

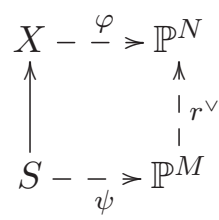

Notice that $\psi$ is dominant since $K_{S}^{2} \leqslant 2$ by assumption. Therefore, $\varphi(S) \subset$ $Y \subset \mathbb{P}^{N}$ is a linear subspace of dimension $\leqslant 2$.

Suppose first that $\operatorname{Bs}\left(-K_{X}\right) \neq \emptyset$. By 5.2, $\operatorname{dim} Y=2$ and $Y \subset \mathbb{P}^{N}$ is a variety of minimal degree. If $K_{S}^{2}=2$, then $Y \subset \mathbb{P}^{N}$ is a plane. But this contradicts 5.1. We conclude that $K_{S}^{2}=1$, and $\varphi(S) \subset Y$ is a line. Notice 
that the $\varphi(S)$ 's yield a covering family of lines on $Y$. By 5.1, there is a rational fibration $s: Y \rightarrow \mathbb{P}^{1}$ by lines such that $f=s \circ \varphi$. Suppose that there is a fibration $g: X \rightarrow \mathbb{P}^{1}$ with general fiber $T$ satisfying $K_{T}^{2} \leqslant 2$ and $g \neq f$. Then, as before, there is a rational fibration $t: Y \rightarrow \mathbb{P}^{1}$ by lines such that $t=t \circ \varphi$ and $t \neq s$. By 5.1 , we must have $Y \cong \mathbb{P}^{1} \times \mathbb{P}^{1} \subset \mathbb{P}^{3}$. It follows that $\varphi=(f, g)$ but this contradicts the fact that $\operatorname{Bs}\left(-K_{X}\right) \neq \emptyset$.

Suppose then that $\operatorname{Bs}\left(-K_{X}\right)=\emptyset$. Notice that $\operatorname{dim} Y=3$. Then we must have $\operatorname{Bs}\left(-K_{S}\right)=\emptyset, K_{S}^{2}=2$, and $h^{0}\left(S, \mathscr{O}_{S}\left(-K_{S}\right)\right)=3$. We claim that the restriction map $r: H^{0}\left(X, \mathscr{O}_{X}\left(-K_{X}\right)\right) \rightarrow H^{0}\left(S, \mathscr{O}_{S}\left(-K_{S}\right)\right)$ is surjective. Suppose otherwise. Then $\left|-K_{S}\right|$ contains a base point free sub-linear system of dimension 1, yielding a contradiction since $-K_{S}$ is ample. We conclude that $\varphi(S) \subset Y \subset \mathbb{P}^{N}$ is a 2-dimensional linear subspace and $\varphi_{\mid S}=\psi$. In particular, $\operatorname{deg}(\varphi)=\operatorname{deg}(\psi)=2$. Therefore, there is a rational fibration $s: Y \rightarrow$ $\mathbb{P}^{1}$ by 2-dimensional linear subspaces, such that $f=s \circ \varphi$. By 5.2 , either $Y \cong \mathbb{P}^{3}$, or $Y \subset \mathbb{P}^{N}$ is a rational normal scroll. If $Y \cong \mathbb{P}^{3}$, then $s: Y \rightarrow \mathbb{P}^{1}$ is a linear projection. Thus $f=s \circ \varphi$ cannot be a morphism, yielding a contradiction. If $Y \subset \mathbb{P}^{N}$ is a rational normal scroll, then $s: Y \rightarrow \mathbb{P}^{1}$ is unique so that $f$ is unique as well. This completes the proof of the lemma.

Example 5.4. Let $S$ be a smooth Del Pezzo surface with $K_{S}^{2} \leqslant 2$, and set $X=S \times \mathbb{P}^{1}$. Denote by $\varphi: X \rightarrow \mathbb{P}^{N}$ the rational map given by the complete linear system $\left|-K_{X}\right|$, and set $Y=\varphi(X)$. Then $Y \cong \mathbb{P}^{1} \times \mathbb{P}^{1}$ (respectively, $Y \cong \mathbb{P}^{2} \times \mathbb{P}^{1}$ ) if $K_{S}^{2}=1$ (respectively, $K_{S}^{2}=2$ ). Any fiber $T \cong S$ of the natural projection $X \rightarrow \mathbb{P}^{1}$ satisfies $K_{T}^{2} \leqslant 2$.

\section{Fano varieties of dimension $\leqslant 3$ with $\operatorname{Nef}(X)=\operatorname{Psef}(X)$}

The classification of Fano manifolds of dimension $\leqslant 3$ with $\operatorname{Nef}(X)=\operatorname{Psef}(X)$ will be presented in this section.

Proposition 6.1. Let $S$ be a locally factorial Del Pezzo surface with $\rho(X) \geqslant$ 2. If $\operatorname{Nef}(S)=\operatorname{Psef}(S)$, then $S \cong \mathbb{P}^{1} \times \mathbb{P}^{1}$.

Proof. Let $\varphi: S \rightarrow \mathbb{P}^{1}$ be an elementary contraction. The irreducible components of fibers of $\varphi$ are smooth rational curves by [3, Corollary 1.9]. Since $S$ is locally factorial, we conclude that $S$ is smooth. The lemma follows easily from the Enriques-Kodaira classification of smooth complex proper surfaces.

The following example shows that the statement of Proposition 6.1 does not hold for normal surfaces with canonical singularities. 
Example 6.2. Let $T$ be the Del Pezzo surface of degree $K_{S}^{2}=4$ with 4 singular points of type $A_{1}$ given by equations

$$
x_{0} x_{1}+x_{2}^{2}=x_{3} x_{4}+x_{2}^{2}=0
$$

in $\mathbb{P}^{4}$. Then $T$ admits a double cover $\mathbb{P}^{1} \times \mathbb{P}^{1} \rightarrow T$ ramified only at the singular points (see $[8$, Proposition 0.4 .2$]$ ). In particular, we have $\operatorname{Nef}(T)=$ $\operatorname{Psef}(T)$.

Fano manifolds with $\operatorname{dim} X=3$ and $\rho(X) \geqslant 2$ were classified by Mori and Mukai in [22]. The classification of those with $\operatorname{Nef}(X)=\operatorname{Psef}(X)$ follows easily.

Theorem 6.3. Let $X$ be a Fano manifold of dimension 3 with $\rho(X) \geqslant 2$. If $\operatorname{Nef}(X)=\operatorname{Psef}(X)$, then $X$ is isomorphic to one of the following.

1) $\mathbb{P}^{1} \times \mathbb{P}^{2}$.

2) A double cover of $\mathbb{P}^{1} \times \mathbb{P}^{2}$ branched along a divisor of type $(2,2)$.

3) A double cover of $\mathbb{P}^{1} \times \mathbb{P}^{2}$ branched along a divisor of type $(2,4)$.

4) $A$ double cover of $Y=\mathbb{P}_{\mathbb{P}^{2}}\left(T_{\mathbb{P}^{2}}\right)$ whose branched locus is a member of $\left|-K_{Y}\right|$.

5) A hypersurface of type $(1,1)$ in $\mathbb{P}^{2} \times \mathbb{P}^{2}$.

6) A hypersurface of type $(1,2)$ in $\mathbb{P}^{2} \times \mathbb{P}^{2}$.

7) A hypersurface of type $(2,2)$ in $\mathbb{P}^{2} \times \mathbb{P}^{2}$.

8) A double cover of $\mathbb{P}^{1} \times \mathbb{P}^{1} \times \mathbb{P}^{1}$ branched along a divisor of type $(2,2,2)$.

\section{Finite morphisms onto $\mathbb{P}^{1} \times \cdots \times \mathbb{P}^{1}$}

In this section we prove Proposition 1.4.

Lemma 7.1. Let $S$ be a $\mathbb{Q}$-Fano surface with Gorenstein singularities, and let $C_{1}$ and $C_{2}$ be two curves contained in the smooth locus of $S$ satisfying $C_{1}^{2}=C_{2}^{2}=0$. Then

$$
2 K_{S}^{2}\left(C_{1} \cdot C_{2}\right) \leqslant\left(K_{S} \cdot C_{1}+K_{S} \cdot C_{2}\right)^{2}
$$

Proof. Let $\mu: T \rightarrow S$ be the minimal resolution of singularities of $S$, and denote by $\tilde{C}_{1}$ and $\tilde{C}_{2}$ the strict transforms in $T$ of $C_{1}$ and $C_{2}$ respectively. 
Using the projection formula, we obtain $\left(\mu^{*} K_{S}\right)^{2}=K_{S}^{2}>0$. Therefore, by the Hodge index theorem, we have

$$
\left(\mu^{*} K_{S}\right)^{2}\left(\tilde{C}_{1}+\tilde{C}_{2}\right)^{2} \leqslant\left(\mu^{*} K_{S} \cdot\left(\tilde{C}_{1}+\tilde{C}_{2}\right)\right)^{2} .
$$

The assumption that $C_{1}$ and $C_{2}$ are contained in the smooth locus of $S$ yields $\left(\mu^{*} K_{S}\right)^{2}\left(\tilde{C}_{1}+\tilde{C}_{2}\right)^{2}=2 K_{S}^{2}\left(C_{1} \cdot C_{2}\right)$. Finally, $\mu^{*} K_{S} \cdot\left(\tilde{C}_{1}+\tilde{C}_{2}\right)=K_{S} \cdot\left(C_{1}+\right.$ $C_{2}$ ) by the projection formula, completing the proof of the lemma.

Proposition 7.2. Let $S$ be a $\mathbb{Q}$-Fano surface with Gorenstein singularities satisfying $K_{S}^{2} \geqslant 3$, and let $f_{1}: S \rightarrow \mathbb{P}^{1}$ and $f_{2}: S \rightarrow \mathbb{P}^{1}$ be two surjective morphisms with connected fibers. Suppose that the induced morphism $f=$ $\left(f_{1}, f_{2}\right): S \rightarrow \mathbb{P}^{1} \times \mathbb{P}^{1}$ is finite. Then either $K_{S}^{2}=4$ and $f$ makes $S$ a double cover of $\mathbb{P}^{1} \times \mathbb{P}^{1}$ branched along a reduced divisor of type $(2,2)$, or $K_{S}^{2}=8$ and $f$ induces an isomorphism $S \cong \mathbb{P}^{1} \times \mathbb{P}^{1}$.

Proof. Let $C_{1}$ and $C_{2}$ be general fibers of $f_{1}$ and $f_{2}$ respectively. Notice that $-K_{S} \cdot C_{1}=-K_{S} \cdot C_{2}=2$. By Lemma 7.1 , we have

$$
6 C_{1} \cdot C_{2} \leqslant 2 K_{S}^{2}\left(C_{1} \cdot C_{2}\right) \leqslant\left(K_{S} \cdot C_{1}+K_{S} \cdot C_{2}\right)^{2}=16,
$$

and thus $\operatorname{deg}(f)=C_{1} \cdot C_{2} \leqslant 2$. If $\operatorname{deg}(f)=1$, then $S \cong \mathbb{P}^{1} \times \mathbb{P}^{1}$ and $K_{S}^{2}=8$. If $\operatorname{deg}(f)=2$, then $f$ makes $S$ a double cover of $\mathbb{P}^{1} \times \mathbb{P}^{1}$ branched along a reduced divisor of type $(2,2)$ by Lemma 3.2 . A straightaforward computation then shows that $K_{S}^{2}=4$.

Proposition 7.3. Let $X$ be a $\mathbb{Q}$-Fano variety of dimension $n \geqslant 3$ with Gorenstein canonical singularities. Let $f: X \rightarrow\left(\mathbb{P}^{1}\right)^{n}$ be a finite morphism of degree $\operatorname{deg}(f)>1$ such that each projection $X \rightarrow\left(\mathbb{P}^{1}\right)^{n-1}$ has connected fibers. Then $f$ makes $X$ a double cover of $\left(\mathbb{P}^{1}\right)^{n}$ branched along a reduced divisor of type $(2, \ldots, 2)$.

Proof. By Lemma 3.2, it suffices to show that $\operatorname{deg}(f)=2$.

Suppose first that $n=3$, and let $f_{1}: X \rightarrow \mathbb{P}^{1}$ and $f^{1}: X \rightarrow \mathbb{P}^{1} \times \mathbb{P}^{1}$ such that $f=\left(f_{1}, f^{1}\right)$. Let $S$ be a general fiber of $f_{1}$. Notice that $S$ is a Del Pezzo surface. Moreover, the restriction of $f^{1}$ to $S$ induces a finite morphism $f^{1}{ }_{\mid S}: S \rightarrow \mathbb{P}^{1} \times \mathbb{P}^{1}$ such that each projection $S \rightarrow \mathbb{P}^{1}$ has connected fibers, and $\operatorname{deg}(f)=\operatorname{deg}\left(f^{1}{ }_{\mid S}\right)$. By Lemma 5.3, we may assume without loss of generality that $K_{S}^{2} \geqslant 3$. We conclude that $\operatorname{deg}(f)=\operatorname{deg}\left(f^{1}{ }_{\mid S}\right)=2$ by Proposition 7.2. This proves the proposition in the case when $n=3$. 
Suppose from now on that $n \geqslant 4$ is arbitrary, and let $f_{1}: X \rightarrow\left(\mathbb{P}^{1}\right)^{n-3}$ and $f^{1}: X \rightarrow\left(\mathbb{P}^{1}\right)^{3}$ such that $f=\left(f_{1}, f^{1}\right)$. Let $F$ be a general fiber of $f_{1}$. Then $F$ is a $\mathbb{Q}$-Fano variety of dimension 3 with Gorenstein canonical singularities. The restriction of $f^{1}$ to $F$ induces a finite morphism $F \rightarrow\left(\mathbb{P}^{1}\right)^{3}$ such that each projection $F \rightarrow\left(\mathbb{P}^{1}\right)^{2}$ has connected fibers, and $\operatorname{deg}(f)=\operatorname{deg}\left(f^{1}\right)$. We conclude that $\operatorname{deg}(f)=\operatorname{deg}\left(f^{1}\right)=2$ from the previous case, completing the proof of the proposition.

Remark 7.4. Proposition 10.4 shows that the statement of Proposition 7.3 above becomes wrong when $\operatorname{dim} X=2$.

Proof of Proposition 1.4. Let $X$ be a $\mathbb{Q}$-Fano variety of dimension $n \geqslant 3$ with Gorenstein canonical singularities. Suppose that $\operatorname{Nef}(X)=\operatorname{Psef}(X)$ and $\rho(X)=n$. Suppose furthermore that each non-trivial contraction $X \rightarrow Y$ with $\operatorname{dim} Y<n$ satisfies $Y \cong \mathbb{P}^{1} \times \cdots \times \mathbb{P}^{1}$.

By Lemma 4.9, the Mori cone $\mathrm{NE}(X)$ is simplicial. Let $V_{1}, \ldots, V_{n}$ be the codimension 1 faces of $\mathrm{NE}(X)$, and denote by $f_{i}: X \rightarrow \mathbb{P}^{1}$ the contraction of $V_{i}$. Set $f=\left(f_{1}, \ldots, f_{n}\right): X \rightarrow\left(\mathbb{P}^{1}\right)^{n}$. Notice that $f$ is a finite morphism. If $\operatorname{deg}(f)=1$, then $X \cong\left(\mathbb{P}^{1}\right)^{n}$. Suppose from now on that $\operatorname{deg}(f)>1$. Let $g: X \rightarrow\left(\mathbb{P}^{1}\right)^{n-1}$ be a projection with Stein factorization $\varphi: X \rightarrow Y \cong$ $\left(\mathbb{P}^{1}\right)^{n-1}$. Then $\varphi$ is given by $n-1$ morphisms with connected fibers $X \rightarrow \mathbb{P}^{1}$, and hence $g=\varphi$. Therefore, Proposition 7.3 applies. Using 3.1, we conclude that $f$ makes $X$ a double cover of $\left(\mathbb{P}^{1}\right)^{n}$ branched along a reduced divisor of type $(2, \ldots, 2)$. This completes the proof of the theorem.

\section{Finite morphisms onto $\mathbb{Q}$-Fano cyclic covers of $\mathbb{P}^{1} \times \cdots \times \mathbb{P}^{1}$}

In this section we prove Theorem 1.3.

Lemma 8.1. Let $f: Z \rightarrow Y$ be a finite surjective morphism of normal projective $\mathbb{Q}$-Gorenstein varieties. If $-K_{Z}$ is big, then so is $-K_{Y}$.

Proof. Denote by $R$ the ramification divisor of $f$. The Hurwitz formula $K_{Z} \sim f^{*} K_{Y}+R$ shows that $-f^{*} K_{Y} \sim-K_{Z}+R$ is big. Let $A$ be an ample Cartier divisor on $Y, E$ an effective divisor on $X$, and $m$ a positive integer such that $m\left(-f^{*} K_{Y}\right) \sim_{\mathbb{Q}} f^{*} A+E$. Then $-K_{Y} \sim_{\mathbb{Q}} \frac{1}{m} A+\frac{1}{m \operatorname{deg}(f)} f_{*} E$ by the projection formula. Hence $-K_{Y}$ is big, proving the lemma.

Lemma 8.2. Let $Z$ be a $\mathbb{Q}$-Fano variety of dimension $n \geqslant 2, Y$ a double cover of $\left(\mathbb{P}^{1}\right)^{n}$ branched along a reduced divisor $B=\sum_{j \in J} B_{j}$ of type 
$(2, \ldots, 2)$, and $f: Z \rightarrow Y$ a finite morphism. Consider a projection $\pi_{1}: Y \rightarrow$ $\mathbb{P}^{1}$, and set $f_{1}=\pi_{1} \circ f: Z \rightarrow \mathbb{P}^{1}$. Suppose that $B_{j}$ is not contracted by $\pi_{1}$ for each $j \in J$. Then $f_{1}$ has connected fibers.

Proof. Denote by $\pi: Y \rightarrow\left(\mathbb{P}^{1}\right)^{n}$ the natural morphism, and let $\pi^{1}: Y \rightarrow$ $\left(\mathbb{P}^{1}\right)^{n-1}$ such that $\pi=\left(\pi_{1}, \pi^{1}\right)$. The Stein factorization $g_{1}: Z \rightarrow \mathbb{P}^{1}$ of $f_{1}=$ $\pi_{1} \circ f$ fits into a commutative diagram

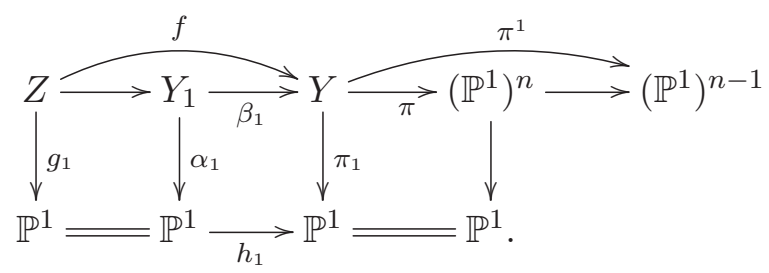

where $\left(\alpha_{1}, \pi_{1} \circ \beta_{1}\right): Y_{1} \rightarrow\left(\mathbb{P}^{1}\right)^{n}$ is the double cover of $\left(\mathbb{P}^{1}\right)^{n}$ branched along $\left(h_{1}, \operatorname{Id}_{\left(\mathbb{P}^{1}\right)^{n-1}}\right)^{*} B$. The variety $Y_{1}$ is Cohen Macaulay by 3.1. All fibers of $\pi_{1}$ are generically reduced since $\pi_{1}$ does not contract any irreducible component of $B$. This implies that all fibers of $\alpha_{1}$ are generically reduced as well, and hence $\operatorname{dim}\left(\operatorname{Sing}\left(Y_{1}\right)\right) \leqslant n-2$. We conclude that $Y_{1}$ is normal. A straightforward computation gives

$$
\mathscr{O}_{Y_{1}}\left(-K_{Y_{1}}\right) \cong \alpha_{1}^{*} \mathscr{O}_{\mathbb{P}^{1}}\left(2-\operatorname{deg}\left(h_{1}\right)\right) \otimes\left(\pi^{1} \circ \beta_{1}\right)^{*}\left(\mathscr{O}_{\mathbb{P}^{1}}(1) \otimes \cdots \otimes \mathscr{O}_{\mathbb{P}^{1}}(1)\right) .
$$

By Lemma 8.1, $-K_{Y_{1}}$ is big. Hence, we must have $\operatorname{deg}\left(h_{1}\right)=1$, proving the lemma.

Proposition 8.3. Let $S$ be a $\mathbb{Q}$-Fano surface with Gorenstein singularities satisfying $K_{S}^{2} \geqslant 3, T$ a double cover of $\mathbb{P}^{1} \times \mathbb{P}^{1}$ branched along a reduced divisor $B=\sum_{j \in J} B_{j}$ of type $(2,2)$, and $f: S \rightarrow T$ a finite morphism. Suppose that $B_{j}$ is ample for each $j \in J$. Then $\operatorname{deg}(f)=1$.

Proof. Denote by $\pi: T \rightarrow \mathbb{P}^{1} \times \mathbb{P}^{1}$ the natural morphism, and denote by $\pi_{1}: T \rightarrow \mathbb{P}^{1}$ and $\pi_{2}: T \rightarrow \mathbb{P}^{1}$ the projections. Set $g_{i}=\pi_{i} \circ f: S \rightarrow \mathbb{P}^{1}$, and $g=\left(g_{1}, g_{2}\right): S \rightarrow \mathbb{P}^{1} \times \mathbb{P}^{1}$ so that $g=\pi \circ f$. By Lemma 8.2 above, $g_{i}$ has connected fibers. By Lemma 7.1 , we have $\operatorname{deg}(g) K_{S}^{2} \leqslant 8$. Since $\operatorname{deg}(g)=$ $2 \operatorname{deg}(f)$, we obtain

$$
\operatorname{deg}(f) \leqslant \frac{4}{K_{S}^{2}} \leqslant \frac{4}{3}
$$

Thus $\operatorname{deg}(f)=1$, proving the proposition. 
The following example shows that the statement of Proposition 8.3 becomes wrong if one relaxes the assumption on $B$.

Example 8.4. Let $C_{1}$ and $C_{2}$ be smooth rational curves, and let $c \in C_{1}$. Denote by $p_{1}$ the projection $C_{1} \times C_{2} \rightarrow C_{1}$, and set $B_{1}=p_{1}^{-1}(c)$. Let $B_{2}$ be a general member in the linear system $\left|\mathscr{O}_{C_{1}}(1) \otimes \mathscr{O}_{C_{2}}(2)\right|$. Set $B=B_{1}+$ $B_{2}$, and let $\pi: S \rightarrow C_{1} \times C_{2}$ be the 2-fold cyclic cover branched along $B$. Then $S$ is a normal surface of degree $K_{S}^{2}=4$ with 2 singular points of type $A_{1}$. Let $c^{\prime} \in C_{1}$ with $c^{\prime} \neq c$, and let $h: \bar{C}_{1} \rightarrow C_{1}$ be the 2 -fold cyclic cover branched along $c+c^{\prime}$. Let $\bar{S}$ be the normalization of the fiber product $S \times{ }_{C_{1}}$ $\bar{C}_{1}$ with natural morphisms $\alpha: \bar{S} \rightarrow \bar{C}_{1}$ and $\beta: \bar{S} \rightarrow S$. A straightforward computation shows that $\beta$ is étale at each point in $\bar{S} \backslash\left((\pi \circ \beta)^{-1}\left(B_{1} \cap B_{2}\right) \cup\right.$ $\left.(h \circ \alpha)^{-1}\left(c^{\prime}\right)\right)$. Moreover, $\beta$ ramifies along $(h \circ \alpha)^{-1}\left(c^{\prime}\right)$ with multiplicity 2 . Therefore, $K_{\bar{S}} \sim \beta^{*} K_{S}+(h \circ \alpha)^{-1}\left(c^{\prime}\right)$. We conclude that $\bar{S}$ is $\mathbb{Q}$-Fano with Gorenstein singularities. Notice that $\pi_{1}(S \backslash \operatorname{Sing}(S)) \cong \pi_{1}\left(\mathbb{P}^{1} \backslash\{c\}\right)=\{1\}$ by Lemma 3.13. Moreover, by Proposition 7.2 , we have $\bar{S} \cong \mathbb{P}^{1} \times \mathbb{P}^{1}$.

In the setup of Proposition 8.3, suppose moreover that $S$ and $T$ are smooth. Then we show that the conclusion still holds when $K_{S}^{2} \leqslant 2$.

Proposition 8.5. Let $S$ and $T$ be smooth Del Pezzo surfaces, and let $f: S \rightarrow T$ be a finite morphism. Suppose that $K_{T}^{2}=4$. Then $\operatorname{deg}(f)=1$.

Proof. By [9, Theorem 8.6.2], $T$ is isomorphic to the complete intersection given by equations

$$
x_{0}^{2}+x_{1}^{2}+x_{2}^{2}+x_{3}^{2}+x_{4}^{2}=a_{0} x_{0}^{2}+a_{1} x_{1}^{2}+a_{2} x_{2}^{2}+a_{3} x_{3}^{2}+a_{4} x_{4}^{2}=0
$$

in $\mathbb{P}^{4}$, where $a_{i} \neq a_{j}$ for $i \neq j$. We may assume without loss of generality that $a_{4} \neq 0$. Thus, the projection from $(0,0,0,0,1)$ induces a finite morphism $\pi: T \rightarrow Q$ of degree 2 onto the smooth quadric surface $Q \cong \mathbb{P}^{1} \times \mathbb{P}^{1}$ given by equation

$$
\left(a_{0}-a_{4}\right) x_{0}^{2}+\left(a_{1}-a_{4}\right) x_{1}^{2}+\left(a_{2}-a_{4}\right) x_{2}^{2}+\left(a_{3}-a_{4}\right) x_{3}^{2}=0 .
$$

Moreover, $\pi$ is branched along a smooth curve of type $(2,2)$. Denote by $\pi_{i}: Y \rightarrow \mathbb{P}^{1}$ with $i \in\{1,2\}$ the natural projections, and set $g_{i}=\pi_{i} \circ f: S \rightarrow$ $\mathbb{P}^{1}$. By Lemma 8.2, $g_{i}$ has connected fibers. Let $F$ be a component of a reducible fiber of $\pi$, and set $E=f^{-1}(F)$. Then $E$ and $F$ are exceptional curves on $S$ and $T$ respectively, and $f^{*} F=E$. Taking squares gives $\operatorname{deg}(f)=$ 1 , proving the proposition. 
Proof of Theorem 1.3. Let $Z$ be a $\mathbb{Q}$-Fano variety of dimension $n \geqslant 3$ with Gorenstein canonical singularities, $Y$ a double cover of $\left(\mathbb{P}^{1}\right)^{n}$ branched along a reduced divisor $B=\sum_{j \in J} B_{j}$ of type $(2, \ldots, 2)$, and $f: Z \rightarrow Y$ a finite morphism. Suppose that $B_{j}$ is ample for each $j \in J$.

Let $p_{1}:\left(\mathbb{P}^{1}\right)^{n} \rightarrow \mathbb{P}^{1}$ be any projection, and let $p^{1}:\left(\mathbb{P}^{1}\right)^{n} \rightarrow\left(\mathbb{P}^{1}\right)^{n-1}$ be the projection onto the product of the remaining factors. Denote by $\pi: Y \rightarrow$ $\left(\mathbb{P}^{1}\right)^{n}$ the natural morphism, and set $\pi_{1}=p_{1} \circ \pi$ and $\pi^{1}=p^{1} \circ \pi$ so that $\pi=\left(\pi_{1}, \pi^{1}\right)$. Let $Y_{1}$ be a general fiber of $\pi_{1}$. Then $\pi_{\mid Y_{1}}$ makes $Y_{1}$ a double cover of $\pi\left(Y_{1}\right) \cong\left(\mathbb{P}^{1}\right)^{n-1}$ branched along the reduced divisor $B_{\mid \pi\left(Y_{1}\right)}$.

We show that there is a dense open subset $U \subset \mathbb{P}^{1}$ with the following property. For any $w \in U$, denote by $Y_{w}$ the fiber of $\pi_{1}$ over $w$. Then any irreducible component of $B_{\mid \pi\left(Y_{w}\right)}$ is ample. To prove the claim, suppose to the contrary that there is a dense subset $W \subset \mathbb{P}^{1}$ such that, for any $w \in W$, some irreducible component of $B_{\mid \pi\left(Y_{w}\right)}$ is not ample. Notice that an effective divisor on $\pi\left(Y_{w}\right)$ is not ample if and only if it is the pullback of some effective divisor under a projection $\pi\left(Y_{w}\right) \cong\left(\mathbb{P}^{1}\right)^{n-1} \rightarrow\left(\mathbb{P}^{1}\right)^{n-2}$. After replacing $W$ with a subset of it if necessary, we may assume that there exists a projection $q^{1}:\left(\mathbb{P}^{1}\right)^{n-1} \rightarrow\left(\mathbb{P}^{1}\right)^{n-2}$ such that, for any $w \in W, B_{\mid \pi\left(Y_{w}\right)}$ contains in its support the pullback of a prime divisor under $q^{1} \circ p_{\mid \pi\left(Y_{w}\right)}^{1}: \pi\left(Y_{w}\right) \rightarrow\left(\mathbb{P}^{1}\right)^{n-2}$. This implies that $B$ contains in its support the pullback of some effective divisor under $\left(p_{1}, q \circ p^{1}\right):\left(\mathbb{P}^{1}\right)^{n} \rightarrow \mathbb{P}^{1} \times\left(\mathbb{P}^{1}\right)^{n-2}$. But this contradicts the fact that $B_{j}$ is ample for each $j \in J$, proving our claim. Thus, we may assume that any irreducible component of $B_{\mid \pi_{1}\left(Y_{1}\right)}$ is ample.

By Lemma 8.2, $Z_{1}=f^{-1}\left(Y_{1}\right)$ is connected. Moreover, $Z_{1}$ has Gorenstein canonical singularities, and the restriction of $f$ to $Z_{1}$ induces a finite morphism $f_{1}: Z_{1} \rightarrow Y_{1}$ with $\operatorname{deg}\left(f_{1}\right)=\operatorname{deg}(f)$. Therefore, to prove the proposition, it suffices to consider the case when $n=3$. By Lemma 5.3, we may assume without loss of generality that $K_{Y_{1}}^{2} \geqslant 3$. Then the result follows from Proposition 8.3.

Corollary 8.6. Let $Z$ be a Fano manifold of dimension $\geqslant 2, Y$ a smooth double cover of $\mathbb{P}^{1} \times \cdots \times \mathbb{P}^{1}$ branched along a reduced divisor $B$ of type $(2, \ldots, 2)$, and $f: Z \rightarrow Y$ a finite morphism. Then $\operatorname{deg}(f)=1$.

Proof. If $\operatorname{dim} Y=2$, then the result follows from Proposition 8.5. Suppose from now on that $\operatorname{dim} Y \geqslant 3$. Since $Y$ is smooth, $\operatorname{Supp}(B)$ is smooth by 3.1, and hence irreducible. Thus Theorem 1.3 applies. 


\section{Proof of Theorem 1.1}

We are now in position to prove our main result. Notice that Theorem 1.1 is an immediate consequence of Lemma 4.5 and Theorem 9.1 below.

Theorem 9.1. Let $(X, B)$ be a $\mathbb{Q}$-Fano pair with locally factorial canonical singularities. Suppose that $\operatorname{Nef}(X)=\operatorname{Psef}(X)$ and $\rho(X)=\operatorname{dim} X$. Then $X \cong X_{1} \times \cdots \times X_{m}$ where $X_{i}$ is a double cover of $\mathbb{P}^{1} \times \cdots \times \mathbb{P}^{1}$ branched along a reduced divisor of type $(2, \ldots, 2)$ for each $i \in\{1, \ldots, m\}$.

Proof. Notice that $B \in \operatorname{Nef}(X)$. Thus $X$ is a $\mathbb{Q}$-Fano variety with locally factorial canonical singularities (see [19, Corollary 2.35]). To prove Theorem 9.1 , we argue by induction on $n=\operatorname{dim} X$. By Lemma 4.9, the Mori cone $\mathrm{NE}(X)$ is simplicial, and $\operatorname{dim} \mathrm{NE}(X)=n$ by assumption.

If $n=2$, then the result follows from Proposition 6.1 .

Suppose that $n \geqslant 3$.

Let $V$ be a face of $\mathrm{NE}(X)$, and denote by $\varphi: X \rightarrow Y$ the corresponding contraction. Then $\varphi$ is equidimensional and $\operatorname{dim} Y=n-\operatorname{dim} V$ by Lemma 4.9. From Lemma 4.2, we infer that $\operatorname{Nef}(Y)=\operatorname{Psef}(Y)$. By Lemma 4.5, there exists an effective $\mathbb{Q}$-divisor $B_{Y}$ on $Y$ such that $\left(Y, B_{Y}\right)$ is $\mathbb{Q}$-Fano with $\log$ terminal singularities. Finally, by Corollary 4.8, $Y$ is locally factorial. Arguing as above, we conclude that $Y$ is a $\mathbb{Q}$-Fano variety with canonical singularities. Hence, $Y$ satisfies the conclusion of Theorem 1.1 provided that $\operatorname{dim} Y<\operatorname{dim} X$, or equivalently $\{0\} \subsetneq V \subsetneq \mathrm{NE}(X)$ by Lemma 4.4.

Suppose first that there is a face $\{0\} \subsetneq V \subsetneq \mathrm{NE}(X)$ such that $Y$ is a double cover of $\left(\mathbb{P}^{1}\right)^{m}$ branched along a divisor $C$ of type $(2, \ldots, 2)$ with $\operatorname{dim} Y=$ $m \geqslant 2$. Notice that $C$ is reduced by 3.1 and irreducible by Lemma 3.7. If $m=2$, then $Y$ is a Del Pezzo surface of degree 4 satisfying $\operatorname{Nef}(Y)=$ $\operatorname{Psef}(Y)$. But this contradicts Proposition 6.1, and therefore, we must have $\operatorname{dim} Y=m \geqslant 3$. Let $W$ be the face of $\mathrm{NE}(X)$ such that $V+W=\mathrm{NE}(X)$ and $\operatorname{dim} W=n-\operatorname{dim} V=m$. Denote by $\psi: X \rightarrow T$ the contraction of $W$, and consider the finite morphism $f=(\varphi, \psi): X \rightarrow Y \times T$. Let $Z$ be a general fiber of $\psi$. Then $Z$ is a $\mathbb{Q}$-Fano variety with Gorenstein canonical singularities, and the restriction of $f$ to $Z$ induces a finite morphism $f_{\mid Z}: Z \rightarrow Y$ with $\operatorname{deg}\left(f_{\mid Z}\right)=\operatorname{deg}(f)$. By Theorem 1.3, we conclude that $\operatorname{deg}\left(f_{\mid Z}\right)=1$. The theorem follows easily in this case.

Suppose now that, for each face $\{0\} \subsetneq V \subsetneq \mathrm{NE}(X)$, we have $Y \cong\left(\mathbb{P}^{1}\right)^{m}$ for some $m \geqslant 1$. Then apply Proposition 1.4 to conclude that, either $X \cong$ $\left(\mathbb{P}^{1}\right)^{n}$, or $X$ is a double cover of $\left(\mathbb{P}^{1}\right)^{n}$ branched along a reduced divisor of type $(2, \ldots, 2)$. This completes the proof of the theorem. 
The following example shows that Theorem 1.1 does not hold for $\mathbb{Q}$-Fano varieties with Gorenstein canonical singularities.

Example 9.2. Fix integers $m \geqslant 2$ and $n \geqslant 2$, and let $\zeta$ be a primitive $m^{\text {th }}$ root of unity. The group $G=\langle\zeta\rangle$ acts on $\mathbb{P}^{1} \times \cdots \times \mathbb{P}^{1}$ by

$$
\zeta \cdot\left(x_{1}, y_{1}\right) \times \cdots \times\left(x_{n}, y_{n}\right)=\left(x_{1}, \zeta y_{1}\right) \times \cdots \times\left(x_{n}, \zeta y_{n}\right) \text {. }
$$

Set $X=\left(\mathbb{P}^{1} \times \cdots \times \mathbb{P}^{1}\right) / G$. Then $X$ has $\mathbb{Q}$-factorial log terminal singularities and Picard number $\rho(X) \leqslant \operatorname{dim} X=n$ by 4 , and satisfies $\mathrm{NE}(X)=$ $\operatorname{Psef}(X)$. Notice that $X$ admits a finite morphism onto $\left(\mathbb{P}^{1} / G\right) \times \cdots \times$ $\left(\mathbb{P}^{1} / G\right) \cong \mathbb{P}^{1} \times \cdots \times \mathbb{P}^{1}$. Thus $\rho(X) \geqslant \operatorname{dim} X$, and hence $\rho(X)=\operatorname{dim} X$. The variety $X$ has isolated singularities and the natural morphism $\mathbb{P}^{1} \times \cdots \times$ $\mathbb{P}^{1} \rightarrow X$ is étale over $X \backslash \operatorname{Sing}(X)$. This implies that $X$ is a $\mathbb{Q}$-Fano variety. We claim that $X$ is not locally factorial. Notice that the open subset $U=\left\{x_{1} \cdots x_{n} \neq 0\right\} \cong \mathbb{A}^{n} \subset \mathbb{P}^{1} \times \cdots \times \mathbb{P}^{1}$ is $G$-stable. Set $W=U / G$ with natural morphism $\pi: U \rightarrow W$. We claim that the ideal sheaf of the reduced hypersurface $H=\pi\left(\left\{y_{1}=0\right\}\right)$ is not locally free at $\pi((0, \ldots, 0))$. Suppose to the contrary that $\mathscr{I}_{H}$ is invertible at $(0, \ldots, 0)$. The functions $y_{1} y_{2}^{m-1}$ and $y_{1}^{m}$ on $U$ are $G$-invariant, and hence there exist functions $g_{1}$ and $g_{2}$ on $W$ such that $y_{1} y_{2}^{m-1}=g_{1} \circ \pi$ and $y_{1}^{m}=g_{2} \circ \pi$. Then $\mathscr{I}_{H,(0, \ldots, 0)}=g_{1} \mathscr{O}_{W,(0, \ldots, 0)}$ and $g_{2} \in \mathscr{I}_{H,(0, \ldots, 0)}^{m}=g_{1}^{m} \mathscr{O}_{W,(0, \ldots, 0)}$ since $y_{1} y_{2}^{m-1}=g_{1} \circ \pi$ and $y_{1}^{m}=g_{2} \circ \pi$ vanish at order 1 and $m$ respectively along $\left\{y_{1}=0\right\}$. This in turn implies that $y_{1}^{m}=g_{2} \circ \pi$ vanishes at order $\geqslant m(m-1)$ along $\left\{y_{2}=0\right\}$, yielding a contradiction. This proves that $X$ is not locally factorial. Finallly, by [26], $X$ is Gorenstein if and only if $n-2 k \equiv 0[m]$ for any $0 \leqslant k \leqslant n$, e.g. $n$ is even and $m=2$.

Suppose that $n \geqslant 3$. Then $X$ does not satisfy the conclusion of Theorem 1.1. To prove the claim, we argue by contradiction, and assume that $X \cong X_{1} \times \cdots \times X_{s}$ where $X_{i}$ is a double cover of $\mathbb{P}^{1} \times \cdots \times \mathbb{P}^{1}$ branched along a reduced divisor of type $(2, \ldots, 2)$ for each $i \in\{1, \ldots, s\}$. Since $X$ has isolated singularities, we must have $s=1$. By Lemma 3.12, we conclude that $\pi_{1}(X \backslash \operatorname{Sing}(X))=\{1\}$, yielding a contradiction since $\pi_{1}(X \backslash \operatorname{Sing}(X)) \cong G$.

The following proposition is a first step towards the classification of Fano manifolds $X$ with $\operatorname{Nef}(X)=\operatorname{Psef}(X)$ and arbitrary Picard number.

Proposition 9.3. Let $(X, B)$ be a $\mathbb{Q}$-Fano pair with locally factorial canonical singularities satisfying $\operatorname{Nef}(X)=\operatorname{Psef}(X)$. Suppose that there is a contraction $\varphi: X \rightarrow Y$ with $\operatorname{dim} Y=\rho(Y)$ and $Y ¥ \mathbb{P}^{1} \times \cdots \times \mathbb{P}^{1}$. Then $X \cong$ 
$Y_{1} \times Y^{1}$ where $Y_{1}$ and $Y^{1}$ are positive dimensional $\mathbb{Q}$-Fano varieties with locally factorial canonical singularities, $\operatorname{Nef}\left(Y_{1}\right)=\operatorname{Psef}\left(Y_{1}\right)$, and $\rho\left(Y_{1}\right)=\operatorname{dim} Y_{1}$.

Proof. Notice that $B \in \operatorname{Nef}(X)$. Therefore, $X$ is a $\mathbb{Q}$-Fano variety with locally factorial canonical singularities. By Lemma 4.5, $\operatorname{Nef}(Y)=\operatorname{Psef}(Y)$, and there exists an effective $\mathbb{Q}$-divisor $B_{Y}$ on $Y$ such that $\left(Y, B_{Y}\right)$ is $\mathbb{Q}$-Fano with $\log$ terminal singularities. From Corollary 4.8, we deduce that $Y$ is locally factorial. Thus Theorem 9.1 applies to $\left(Y, B_{Y}\right)$. We have $Y \cong Y_{1} \times \cdots \times Y_{m}$ where $Y_{i}$ is a double cover of $\mathbb{P}^{1} \times \cdots \times \mathbb{P}^{1}$ branched along a reduced divisor $B_{i}$ of type $(2, \ldots, 2)$ for all $1 \leqslant i \leqslant m$. Since $Y \not \mathbb{P}^{1} \times \cdots \times \mathbb{P}^{1}$, we may assume without loss of generality that $\operatorname{dim} Y_{1} \geqslant 2$. From Corollary 4.8 applied to $X \rightarrow Y_{1}$, we deduce that $Y_{1}$ is locally factorial. Therefore, by Proposition 6.1 , we must have $\operatorname{dim} Y_{1} \geqslant 3$. Notice that $B_{1}$ is irreducible by Lemma 3.7.

By Lemma 4.12 applied to $\varphi_{1}: X \rightarrow Y_{1}$, there exists a contraction $\varphi^{1}$ : $X \rightarrow Y^{1}$ such that the induced morphism $\varphi=\left(\varphi_{1}, \varphi^{1}\right): X \rightarrow Y_{1} \times Y^{1}$ is surjective and finite. Let $Z$ be a general fiber of $\varphi^{1}$. Then $Z$ is a $\mathbb{Q}$-Fano variety with Gorenstein canonical singularities. Moreover, the restriction of $\varphi_{1}$ to $Z$ induces a finite morphism $f: Z \rightarrow Y_{1}$ with $\operatorname{deg}(f)=\operatorname{deg}(\varphi)$. From Theorem 1.3, we conclude that $\operatorname{deg}(\varphi)=1$, completing the proof of the proposition.

Remark 9.4. In the setup of Proposition 9.3, $Y_{1}$ satisfies the conclusion of Theorem 1.1.

\section{Finite morphisms between smooth Del Pezzo surfaces}

In order to prove Theorem 1.1, we were led to study finite morphisms between $\mathbb{Q}$-Fano varieties. In this section we address finite morphisms between smooth Del Pezzo surfaces. We believe that these results are interesting on their own and that they will be useful when considering Fano manifolds with $\operatorname{Nef}(X)=\operatorname{Psef}(X)$ and arbitrary Picard number.

Beauville classified smooth Del Pezzo surfaces which admit an endomorphism of degree $>1$ in [4]. We will consider a finite morphism $f: S \rightarrow T$ of degree $>1$ between smooth Del Pezzo surfaces $S$ and $T$ with $K_{S}^{2} \neq K_{T}^{2}$, or equivalently $K_{S}^{2}<K_{T}^{2}$. We will show that we must have $K_{T}^{2} \geqslant 8$ (see Theorem 1.6). A smooth Del Pezzo surface of degree 8 (respectively, 9) is isomorphic to $\mathbb{P}^{1} \times \mathbb{P}^{1}$ or to $\mathbb{P}^{2}$ blown up at one point (respectively, $\left.\mathbb{P}^{2}\right)$ ). Notice that any projective surface admits a finite morphism of degree $>1$ onto $\mathbb{P}^{2}$ by Noether Normalization Lemma. The classification of smooth Del 
Pezzo surfaces which admit a finite morphism of degre $>1$ onto $\mathbb{P}^{1} \times \mathbb{P}^{1}$ follows easily from Proposition 10.4.

10.1 (Smooth Del Pezzo surfaces). It is well known that a smooth Del Pezzo surface is either $\mathbb{P}^{1} \times \mathbb{P}^{1}$ or the blow up of $\mathbb{P}^{2}$ at $1 \leqslant r \leqslant 8$ points in general position, namely, no three points on a line, no six points on a conic, and if $r=8$, not all of them on a cubic with one of the point being a singular point.

We will make use of the following elementary observation.

Lemma 10.2. Let $S ¥ \mathbb{P}^{1} \times \mathbb{P}^{1}$ be a smooth Del Pezzo surface, and let $f_{1}: S \rightarrow \mathbb{P}^{1}$ be a conic bundle structure on $S$. Set $r=9-K_{S}^{2}$. Then there are $r$ points in general position $p_{1}, \ldots, p_{r}$ in $\mathbb{P}^{2}$ such that the following holds. The surface $S$ is the blow up of $\mathbb{P}^{2}$ in $p_{1}, \ldots, p_{r}$ and $f_{1}$ is induced by the pencil of lines in $\mathbb{P}^{2}$ passing through $p_{1}$.

Proof. Notice that $\rho(S)=10-K_{S}^{2}=r+1$. Let $\mu: S \rightarrow S_{1}$ be a minimal model of $f_{1}: S \rightarrow \mathbb{P}^{1}$, and denote the exceptional prime curves of $\mu$ by $E_{1}, \ldots, E_{r-1}$. Then $S_{1} \cong \mathbb{F}_{m}$ for some integer $m \geqslant 0$, and $f_{1}$ is induced by the natural morphism $\mathbb{F}_{m} \rightarrow \mathbb{P}^{1}$. Let $\sigma_{1} \subset S_{1}$ be a minimal section, and let $\sigma$ be the strict transform of $\sigma_{1}$ in $S$. Then $\sigma^{2} \leqslant \sigma_{1}^{2}=-m$, and hence $m \leqslant 1$ since the only curves with negative square on a smooth Del Pezzo surface are the exceptional ones.

Suppose first that $S_{1} \cong \mathbb{F}_{1}$. Notice that $\sigma_{1} \cap \mu\left(E_{i}\right)=\emptyset$ for all $1 \leqslant i \leqslant$ $r-1$. Consider the blow down $\nu: S_{1} \rightarrow \mathbb{P}^{2}$ of $\sigma_{1}$, and set $\mu_{1}=\nu \circ \mu: S \rightarrow \mathbb{P}^{2}$. Then $S$ is obtained by blowing up $\mu_{1}\left(E_{1}\right), \ldots, \mu_{1}\left(E_{r-1}\right)$, and $\nu\left(\sigma_{1}\right)$ in $\mathbb{P}^{2}$, and $f_{1}$ is induced by the pencil of lines in $\mathbb{P}^{2}$ passing through $\nu\left(\sigma_{1}\right)$.

Suppose now that $S_{1} \cong \mathbb{P}^{1} \times \mathbb{P}^{1}$. Since $S \neq \mathbb{P}^{1} \times \mathbb{P}^{1}$, we must have $r \geqslant 2$. Let $S_{0}$ be the blow up of $S_{1}$ at $\mu\left(E_{1}\right)$, and let $F_{1}, F_{2}$ and $F_{3}$ be the exceptional curves on $S_{0}$. Up to renumbering the $F_{i}$ 's if necessary, we may assume that $F_{1}$ is contracted by the natural morphism $S_{0} \rightarrow S_{1}$. Let $\nu: S_{0} \rightarrow \mathbb{P}^{2}$ be the blow down of $F_{2}$ and $F_{3}$. Denote by $\mu_{1}: S \rightarrow \mathbb{P}^{2}$ the natural morphism. Then $S$ is obtained by blowing up $\mu_{1}\left(E_{2}\right), \ldots, \mu_{1}\left(E_{r-1}\right), \nu\left(F_{2}\right)$, and $\nu\left(F_{3}\right)$ in $\mathbb{P}^{2}$. Moreover, up to renumbering the $F_{i}$ 's if necessary, $f_{1}$ is induced by the pencil of lines in $\mathbb{P}^{2}$ passing through $\nu\left(F_{2}\right)$.

Lemma 10.3. Let $S$ be a smooth Del Pezzo surface of degree $K_{S}^{2}=2$, and let $f_{1}: S \rightarrow \mathbb{P}^{1}$ and $f_{2}: S \rightarrow \mathbb{P}^{1}$ be two conic bundle structures on $S$. Suppose that $f=\left(f_{1}, f_{2}\right): S \rightarrow \mathbb{P}^{1} \times \mathbb{P}^{1}$ is finite. Then there exist 7 points $p_{1}, \ldots, p_{7}$ in general position in $\mathbb{P}^{2}$ such that $S$ is the blow up of $\mathbb{P}^{2}$ at $p_{1}, \ldots, p_{7}$, 
and such that $f_{1}$ is induced by the pencil of lines in $\mathbb{P}^{2}$ passing through $p_{1}$. Moreover, one of the following holds.

- $f_{2}$ is induced by the pencil of cubics passing through $p_{2}, \ldots, p_{7}$ with a point of multiplicity 2 at $p_{7}$, and $\operatorname{deg}(f)=3$.

- $f_{2}$ is induced by the pencil of quartics passing through $p_{1}, \ldots, p_{7}$ with a point of multiplicity 2 at $p_{5}, p_{6}$, and $p_{7}$, and $\operatorname{deg}(f)=3$.

- $f_{2}$ is induced by the pencil of quintics passing through $p_{1}, \ldots, p_{7}$ with a point of multiplicity 2 at 6 out of $p_{1}, \ldots, p_{7}$, and either $\operatorname{deg}(f)=4$ or $\operatorname{deg}(f)=3$ according to whether or not $p_{1}$ is a multiple point.

Conversely, given $S, f_{1}$, and $f_{2}$ as above, $f=\left(f_{1}, f_{2}\right)$ is a finite morphism.

Proof. By Lemma 10.2, there exist 7 points $p_{1}, \ldots, p_{7}$ in general position in $\mathbb{P}^{2}$ such that $S$ is the blow up of $\mathbb{P}^{2}$ at $p_{1}, \ldots, p_{7}$, and such that $f_{1}$ is induced by the pencil of lines in $\mathbb{P}^{2}$ passing through $p_{1}$. The reducible fibers of $f_{1}$ are the reducible curves $\ell_{i} \cup F_{i}$ for $i=2, \ldots, 7$, where $\ell_{i}$ denotes the strict transform in $S$ of the line connecting $p_{1}$ and $p_{i}$, and $F_{1}, \ldots, F_{7}$ denote the exceptional curves in $S$ over the $p_{i}$ 's. By [9, 8.7.1, p. 454], up to renumbering the $p_{i}$ 's if necessary, one of the following holds.

1) $f_{2}$ is induced by the pencil of lines passing through $p_{2}$.

2) $f_{2}$ is induced by the pencil of conics passing through 4 out of $p_{1}, \ldots, p_{5}$.

3) $f_{2}$ is induced by the pencil of cubics passing through $p_{1}, \ldots, p_{6}$ with a point of multiplicity 2 at $p_{1}$ or $p_{2}$.

4) $f_{2}$ is induced by the pencil of cubics passing through $p_{2}, \ldots, p_{7}$ with a point of multiplicity 2 at $p_{7}$.

5) $f_{2}$ is induced by the pencil of quartics passing through $p_{1}, \ldots, p_{7}$ with a point of multiplicity 2 at $p_{1}, p_{2}$, and $p_{3}$.

6) $f_{2}$ is induced by the pencil of quartics passing through $p_{1}, \ldots, p_{7}$ with a point of multiplicity 2 at $p_{5}, p_{6}$, and $p_{7}$.

7) $f_{2}$ is induced by the pencil of quintics passing through $p_{1}, \ldots, p_{7}$ with a point of multiplicity 2 at 6 out of $p_{1}, \ldots, p_{7}$.

In cases (1), (3), and (5), $f_{1}$ and $f_{2}$ contract $\ell_{2}$. This contradicts the fact that $f$ is finite. In case $(2), f_{1}$ and $f_{2}$ contract $F_{6}$, yielding again a contradiction. Therefore, $f_{2}$ satisfies one of the conditions in the statement of the lemma. 
Conversely, let $S$ be the blow-up of $\mathbb{P}^{2}$ in 7 points $p_{1}, \ldots, p_{7}$ in $\mathbb{P}^{2}$ in general position. Let $f_{1}$ be the conic bundle structure on $S$ induced by the pencil of lines in $\mathbb{P}^{2}$ passing through $p_{1}$, and let $f_{2}$ be a conic bundle structure on $S$ satisfying any of the conditions in the statement of the lemma. Set $f=\left(f_{1}, f_{2}\right): S \rightarrow \mathbb{P}^{1} \times \mathbb{P}^{1}$. To prove that $f$ is finite, we have to check that there is no curve on $S$ contracted by both $f_{1}$ and $f_{2}$. Denote by $C\left(m, m_{i_{1}} p_{i_{1}}, \ldots, m_{i_{s}} p_{i_{s}}\right)$ the strict transform in $S$ of an integral plane curve of degree $m$ passing through $p_{i_{1}}, \ldots, p_{i_{s}}$ with multiplicities $m_{i_{1}}, \ldots, m_{i_{s}}$ respectively, where $\left\{i_{1}, \ldots, i_{s}\right\} \subset\{1, \ldots, 7\}$.

Suppose first that we are in case (4) in the statement of Lemma 10.3. Then the reducible fibers of $f_{2}$ are the curves $C\left(3, p_{1}, \ldots, p_{6}, 2 p_{7}\right) \cup F_{1}$ and $C\left(2, p_{i}, p_{j}, p_{k}, p_{l}, p_{7}\right) \cup C\left(1, p_{m}, p_{7}\right)$ where $\{i, j, k, l, m\}=\{2,3,4,5,6\}$. It follows that $f$ is finite in this case.

Suppose now that we are in case (6) in the statement of Lemma 10.3. Then the reducible fibers of $f_{2}$ are the curves $C\left(3, p_{1}, \ldots, p_{4}, 2 p_{i}, p_{j}, p_{k}\right) \cup$ $C\left(1, p_{j}, p_{k}\right)$ where $\{i, j, k\}=\{5,6,7\}$, together with the curves $C\left(2, p_{i}, p_{j}\right.$, $\left.p_{5}, p_{6}, p_{7}\right) \cup C\left(2, p_{k}, p_{l}, p_{5}, p_{6}, p_{7}\right)$ where $\{i, j, k, l\}=\{1,2,3,4\}$. We conclude again that $f$ is finite.

Finally, suppose that we are in case (7) in the statement of Lemma 10.3. Namely, $f_{2}$ corresponds to the pencil of quintics passing through $p_{1}, \ldots, p_{7}$ with a point of multiplicity 2 at $p_{i_{1}}, \ldots, p_{i_{6}}$ where $\left\{i_{1}, \ldots, i_{6}\right\} \subset\{1, \ldots, 7\}$. Then the reducible fibers of $f_{2}$ are the curves $C\left(3,2 p_{j_{1}}, p_{j_{2}}, \ldots, p_{j_{6}}, p_{7}\right) \cup$ $C\left(2, p_{j_{2}}, \ldots, p_{j_{6}}, p_{7}\right)$ where $\left\{j_{1}, \ldots, j_{6}\right\}=\left\{i_{1}, \ldots, i_{6}\right\}$. We infer that $f$ is finite in this case too, proving the lemma.

Proposition 10.4. Let $S$ be a smooth Del Pezzo surface. Then there exists a finite morphism $S \rightarrow \mathbb{P}^{1} \times \mathbb{P}^{1}$ if and only if $K_{S}^{2} \in\{1,2,4\}$, or $K_{S}^{2}=8$ and $S \cong \mathbb{P}^{1} \times \mathbb{P}^{1}$.

Proof. Suppose first that there exists a finite morphism $f: S \rightarrow \mathbb{P}^{1} \times \mathbb{P}^{1}$. Denote by $f_{1}: S \rightarrow \mathbb{P}^{1}$ and $f_{2}: S \rightarrow \mathbb{P}^{1}$ the natural projections. By replacing $f_{i}$ with its Stein factorization, we may assume that it has connected fibers. The result then follows from Proposition 7.2.

Conversely, let $S$ be a smooth Del Pezzo surface with $K_{S}^{2} \in\{1,2,4\}$.

If $K_{S}^{2}=4$, then $S$ is a double cover of $\mathbb{P}^{1} \times \mathbb{P}^{1}$ (see proof of Proposition 8.5).

Suppose that $K_{S}^{2} \leqslant 2$. By Lemma 10.3, it suffices to consider the case when $K_{S}^{2}=1$. Recall that $S$ is obtained by blowing up 8 points in general position $p_{1}, \ldots, p_{8}$ in $\mathbb{P}^{2}$. Denote by $F_{1}, \ldots, F_{8}$ the exceptional curves in $S$ over the $p_{i}$ 's, and by $C\left(m, m_{i_{1}} p_{i_{1}}, \ldots, m_{i_{s}} p_{i_{s}}\right)$ the strict transform in $S$ 
of an integral plane curve of degree $m$ passing through $p_{i_{1}}, \ldots, p_{i_{s}}$ with multiplicities $m_{i_{1}}, \ldots, m_{i_{s}}$ respectively, where $\left\{i_{1}, \ldots, i_{s}\right\} \subset\{1, \ldots, 8\}$.

Let $f_{1}$ be the conic bundle structure on $S$ induced by the pencil of lines in $\mathbb{P}^{2}$ passing through $p_{1}$, and let $f_{2}$ be the conic bundle structure on $S$ induced by the pencil of plane quartics through $p_{2}, \ldots, p_{8}$ with multiplicity 2 at $p_{6}, p_{7}$ and $p_{8}$. Set $f=\left(f_{1}, f_{2}\right): S \rightarrow \mathbb{P}^{1} \times \mathbb{P}^{1}$. To show that $f$ is finite, we have to check that there is no curve on $S$ contracted by both $f_{1}$ and $f_{2}$. The reducible fibers of $f_{1}$ are the curves $C\left(1, p_{1}, p_{i}\right) \cup F_{i}$ where $i \in\{2, \ldots, r\}$. The reducible fibers of $\pi_{2}$ are the curves $C\left(3, p_{2}, \ldots, p_{5}, 2 p_{i}, p_{j}, p_{k}\right) \cup C\left(1, p_{j}, p_{k}\right)$ where $\{i, j, k\}=\{6,7,8\}$, together with the curves $C\left(2, p_{i}, p_{j}, p_{6}, p_{7}, p_{8}\right) \cup$ $C\left(2, p_{k}, p_{l}, p_{6}, p_{7}, p_{8}\right)$ where $\{i, j, k, l\}=\{2,3,4,5\}$, and $C\left(4, p_{1}, \ldots, p_{5}, 2 p_{6}\right.$, $\left.2 p_{7}, 2 p_{8}\right) \cup F_{1}$. We conclude that $f$ is finite, completing the proof of the proposition.

10.5. Let $S$ and $T$ be smooth Del Pezzo surfaces, and let $f: S \rightarrow T$ be a finite morphism. Let $F$ be an exceptional curve on $T$, and consider the blow down $\nu: T \rightarrow N$ of $F$. Let $\mu: S \rightarrow M$ be the Stein factorization of the composite map $S \rightarrow T \rightarrow N$, and let $g: M \rightarrow N$ be the induced morphism. Because of the genus formula $C^{2}+C \cdot K_{T}=2 p_{a}(C)-2$, the only curves with negative square on $T$ are the exceptional ones. By the Hodge index theorem, we conclude that $f^{-1}(F)$ is the (disjoint) union of exceptional curves. Thus $M$ and $N$ are smooth Del Pezzo surfaces, and $g$ is a finite morphism with $\operatorname{deg}(g)=\operatorname{deg}(f)$.

We now classify endomorphisms of smooth Del Pezzo surfaces of degree 7 .

Lemma 10.6. Let $S$ be the Del Pezzo surface given by blowing up $\mathbb{P}^{1} \times \mathbb{P}^{1}$ at $p \in \mathbb{P}^{1} \times \mathbb{P}^{1}$, and let $f: S \rightarrow S$ be an endomorphism. Then there exist a positive integer $m$ and a choice of coordinates such that $p=(0,1) \times(0,1)$, and either $f$ is given by $\left(x_{1}, x_{2}\right) \times\left(y_{1}, y_{2}\right) \mapsto\left(x_{1}^{m}, x_{2}^{m}\right) \times\left(y_{1}^{m}, y_{2}^{m}\right)$, or $f$ is given by $\left(x_{1}, x_{2}\right) \times\left(y_{1}, y_{2}\right) \mapsto\left(y_{1}^{m}, y_{2}^{m}\right) \times\left(x_{1}^{m}, x_{2}^{m}\right)$.

Proof. Denote by $\nu: S \rightarrow \mathbb{P}^{1} \times \mathbb{P}^{1}$ the natural morphism, with exceptional locus F. By 10.5 and Proposition 10.4, there is a commutative diagram

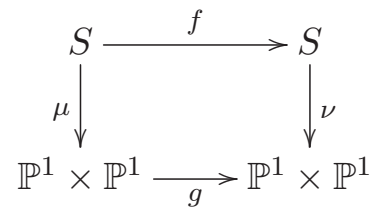


where $g$ is a finite morphism. There exist finite morphisms $g_{1}: \mathbb{P}^{1} \rightarrow \mathbb{P}^{1}$ and $g_{2}: \mathbb{P}^{1} \rightarrow \mathbb{P}^{1}$ such that $g$ is given by $q=\left(q_{1}, q_{2}\right) \mapsto\left(g_{1}\left(q_{1}\right), g_{2}\left(q_{2}\right)\right)$, or $q=\left(q_{1}, q_{2}\right) \mapsto\left(g_{2}\left(q_{2}\right), g_{1}\left(q_{1}\right)\right)$.

Recall that there are 3 exceptional curves $F, F_{1}$ and $F_{2}$ on $S$. Moreover, $F_{1} \cap F_{2}=\emptyset$ and $F \cdot F_{1}=F \cdot F_{2}=1$. This implies that $f^{*} F=\delta F$ for some positive integer $\delta$. In particular, we have $g^{-1}(p)=\{p\}$. Therefore, there exist positive integers $l$ and $m$ and a choice of coordinates such that $p=(0,1) \times$ $(0,1)$, and such that $g_{1}$ and $g_{2}$ are given by $\left(x_{1}, x_{2}\right) \mapsto\left(x_{1}^{l}, x_{2}^{l}\right)$ and $\left(y_{1}, y_{2}\right) \mapsto$ $\left(y_{1}^{m}, y_{2}^{m}\right)$ respectively. Thus $g^{-1} \mathscr{I}_{p} \cdot \mathscr{O}_{\mathbb{P}^{1} \times \mathbb{P}^{1}, p}=\left(\left(\frac{x_{1}}{x_{2}}\right)^{l},\left(\frac{y_{1}}{y_{2}}\right)^{m}\right) \mathscr{O}_{\mathbb{P}^{1} \times \mathbb{P}^{1}, p}$. But $(g \circ \mu)^{-1} \mathscr{I}_{p} \cdot \mathscr{O}_{S} \cong \mathscr{O}_{S}(-\delta F)$ is a line bundle on $S$. A straightforward computation then shows that we must have $l=m$.

We are now ready to prove the main result of this section.

Proof of Theorem 1.6. Let $S$ and $T$ be smooth Del Pezzo surfaces with $K_{S}^{2}<$ $K_{T}^{2}$, and let $f: S \rightarrow T$ be a finite morphism. To prove Theorem 1.6, we argue by contradiction, and assume that $K_{T}^{2} \leqslant 7$.

Step 1. Suppose first that $K_{T}^{2}=7$. Then $T$ is the blow up of $\mathbb{P}^{1} \times \mathbb{P}^{1}$ at some point $p=\left(p_{1}, p_{2}\right) \in \mathbb{P}^{1} \times \mathbb{P}^{1}$. Denote by $\nu: S \rightarrow \mathbb{P}^{1} \times \mathbb{P}^{1}$ the natural morphism, with exceptional locus $F$. By 10.5, there is a commutative diagram

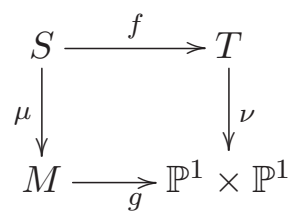

where $M$ is a smooth Del Pezzo surface with $K_{M}^{2}>K_{S}^{2}$, and $g$ is a finite morphism with $\operatorname{deg}(g)=\operatorname{deg}(f)$. Denote by $g_{i}: M \rightarrow \mathbb{P}^{1}$ with $i \in\{1,2\}$ the natural projections, and denote by $h_{i}: M \rightarrow \mathbb{P}^{1}$ their Stein factorizations. There exist endomorphisms $u_{i}: \mathbb{P}^{1} \rightarrow \mathbb{P}^{1}$ such that $g_{i}=u_{i} \circ h_{i}$. Set $u=$ $\left(u_{1}, u_{2}\right): \mathbb{P}^{1} \times \mathbb{P}^{1} \rightarrow \mathbb{P}^{1} \times \mathbb{P}^{1}$ and $h=\left(h_{1}, h_{2}\right): M \rightarrow \mathbb{P}^{1} \times \mathbb{P}^{1}$ so that $g=h \circ$ $u$.

We show that

$$
\# u_{1}^{-1}\left(p_{1}\right)=\# u_{2}^{-1}\left(p_{2}\right)=\# g^{-1}(p)=1
$$

Suppose that $\# u_{1}^{-1}\left(p_{1}\right)<\# g^{-1}(p)$. Notice that $g^{-1}(p) \subset g_{1}^{-1}\left(p_{1}\right)$, and hence $h_{1}\left(g^{-1}(p)\right) \subset h_{1}\left(g_{1}^{-1}\left(p_{1}\right)\right)=u_{1}^{-1}\left(p_{1}\right)$. Thus, there exist $m_{1} \neq m_{2}$ on $M$ with $h_{1}\left(m_{1}\right)=h_{1}\left(m_{2}\right)$ (and $g\left(m_{1}\right)=g\left(m_{2}\right)=p$ ). The fiber $h_{1}^{-1}\left(h_{1}\left(m_{1}\right)\right)$ is either a smooth connected curve with self intersection zero or the union of two exceptional curves on $M$. Hence, $\mu^{-1}\left(h_{1}^{-1}\left(h_{1}\left(m_{1}\right)\right)\right)$ contains a smooth rational 
curve with self intersection $\leqslant-2$, yielding a contradiction. This proves that $\# u_{1}^{-1}\left(p_{1}\right) \geqslant \# g^{-1}(p)$. Similarly, we have $\# u_{2}^{-1}\left(p_{2}\right) \geqslant \# g^{-1}(p)$, and thus

$$
\# g^{-1}(p) \geqslant \# u^{-1}(p)=\# u_{1}^{-1}\left(p_{1}\right) \# u_{2}^{-1}\left(p_{2}\right) \geqslant\left(\# g^{-1}(p)\right)^{2} \text {. }
$$

This implies that $\# u_{1}^{-1}\left(p_{1}\right)=\# u_{2}^{-1}\left(p_{2}\right)=\# g^{-1}(p)=1$, proving $(10.1)$. Set $q=g^{-1}(p)$.

By (10.1), we must have $K_{M}^{2}=K_{S}^{2}+1$, and hence $2 \leqslant K_{M}^{2}=K_{S}^{2}+$ $1<K_{T}^{2}+1=8$. Thus $K_{M}^{2} \in\{2,4\}$ by Proposition 10.4. This implies that $\operatorname{deg}(h) \geqslant 2$.

Since $\# u_{1}^{-1}\left(p_{1}\right)=\# u_{2}^{-1}\left(p_{2}\right)=1$, there exist positive integers $l$ and $m$ and a choice of coordinates such that $p_{1}=(0,1)$ and $p_{2}=(0,1)$, and such that $u_{1}$ and $u_{2}$ are given by $\left(x_{1}, x_{2}\right) \mapsto\left(x_{1}^{l}, x_{2}^{l}\right)$ and $\left(y_{1}, y_{2}\right) \mapsto\left(y_{1}^{m}, y_{2}^{m}\right)$ respectively. Denote the exceptional prime curve of $\mu$ by $E$. We have $f^{*} F=\delta E$ for some positive integer $\delta$. Taking squares gives $\operatorname{deg}(f)=\delta^{2}$. Since $g=$ $h \circ u$, we obtain

$$
\delta^{2}=\operatorname{deg}(f)=\operatorname{deg}(g)=\operatorname{deg}(h) l m .
$$

Notice that the multiplicity of $x_{1} \circ h_{1}$ (respectively, $y_{1} \circ h_{2}$ ) at $q$ is $\leqslant 2$. Since $(g \circ \mu)^{-1} \mathscr{I}_{p} \cdot \mathscr{O}_{S} \cong \mathscr{O}_{S}(-\delta F)$, a straightforward computation shows that

$$
\delta \in\{l, 2 l\} \cap\{m, 2 m\} .
$$

We may assume without loss of generality that $l \leqslant m$. From (10.2) and (10.3), we obtain that either $\delta=m=2 l$ and $\operatorname{deg}(h)=2$, or $\frac{\delta}{2}=m=l$ and $\operatorname{deg}(h)=4$.

Suppose first that $K_{M}^{2}=4$. By Proposition 7.2, we must have $\operatorname{deg}(h)=$ 2 , and $\mathscr{O}_{M}\left(-K_{M}\right) \cong h^{*}\left(\mathscr{O}_{\mathbb{P}^{1}}(1) \otimes \mathscr{O}_{\mathbb{P}^{1}}(1)\right)$. Therefore, $\delta=m=2 l$. A straightforward computation gives $\mathscr{O}_{S}\left(-m K_{S}\right) \cong f^{*}\left(\nu^{*}\left(\mathscr{O}_{\mathbb{P}^{1}}(2) \otimes \mathscr{O}_{\mathbb{P}^{1}}(1)\right) \otimes \mathscr{O}_{T}(-F)\right)$, yielding a contradiction since $\nu^{*}\left(\mathscr{O}_{\mathbb{P}^{1}}(2) \otimes \mathscr{O}_{\mathbb{P}^{1}}(1)\right) \otimes \mathscr{O}_{T}(-F)$ is not an ample line bundle.

Suppose now that $K_{M}^{2}=2$. By Lemma 10.3, we must have $\operatorname{deg}(h) \in$ $\{3,4\}$. Thus, $\operatorname{deg}(h)=4$ and $\frac{\delta}{2}=m=l$. Moreover, $\mathscr{O}_{M}\left(-2 K_{M}\right) \cong$ $h^{*}\left(\mathscr{O}_{\mathbb{P}^{1}}(1) \otimes \mathscr{O}_{\mathbb{P}^{1}}(1)\right)$. A straightforward computation gives $\mathscr{O}_{S}\left(-2 m K_{S}\right) \cong$ $f^{*}\left(\nu^{*}\left(\mathscr{O}_{\mathbb{P}^{1}}(1) \otimes \mathscr{O}_{\mathbb{P}^{1}}(1)\right) \otimes \mathscr{O}_{T}(-F)\right)$. Again, this yields a contradiction since $\nu^{*}\left(\mathscr{O}_{\mathbb{P}^{1}}(1) \otimes \mathscr{O}_{\mathbb{P}^{1}}(1)\right) \otimes \mathscr{O}_{T}(-F)$ is not an ample line bundle.

Step 2. Suppose that $K_{T}^{2} \leqslant 6$.

Let $N$ be the Del Pezzo surface with $K_{N}^{2}=7$ given by blowing up $\mathbb{P}^{1} \times \mathbb{P}^{1}$ at $(0,0) \in \mathbb{P}^{1} \times \mathbb{P}^{1}$, and let $\nu: T \rightarrow N$ be any birational morphism. Denote 
the exceptional prime curves of $\nu$ by $F_{1}, \ldots, F_{k}$, and set $p_{i}=\nu_{1}\left(F_{i}\right)$. By 10.5, there is a commutative diagram

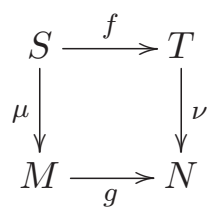

where $M$ is a smooth Del Pezzo surface with $K_{M}^{2}>K_{S}^{2}$, and $g$ is a finite morphism with $\operatorname{deg}(g)=\operatorname{deg}(f)$. By Step 1 , we must have $M \cong N$. Let $R$ be the ramification divisor of $g$. By Lemma 10.6, $g(R)=\sum_{i=0}^{4} R_{i}$ where $R_{0}$ is the exceptional curve of $\varepsilon: N \rightarrow \mathbb{P}^{1} \times \mathbb{P}^{1}, R_{1}$ and $R_{2}$ are the strict transforms in $N$ of the curves $\{0\} \times \mathbb{P}^{1}$ and $\mathbb{P}^{1} \times\{0\}$ respectively, and $R_{3}$ and $R_{4}$ are the strict transforms in $N$ of the curves $\{\infty\} \times \mathbb{P}^{1}$ and $\mathbb{P}^{1} \times\{\infty\}$ for some point $\infty \neq 0$ in $\mathbb{P}^{1}$. Moreover, we have $R=g^{-1}(g(R)) \cong g(R)$.

Fix $i \in\{1, \ldots, k\}$. We claim that $p_{i} \in\left(N \backslash \operatorname{Supp}\left(R_{0}+R_{1}+R_{2}\right)\right) \cup$ $\left(R_{3} \cap R_{4}\right)$. Notice that $R_{0}, R_{1}, R_{2}$ are exceptional curves on $N$, and hence $p_{i} \in N \backslash \operatorname{Supp}\left(R_{0}+R_{1}+R_{2}\right)$. Suppose that $p_{i} \in R_{3} \backslash \operatorname{Supp}\left(R_{2}+R_{4}\right)$ and let $q_{i} \in g^{-1}\left(p_{i}\right)$. Then there is a choice of local coordinates at $q_{i}$ and $p_{i}$ such that $q_{i}=(0,0)$ and $g$ is given by $(x, y) \mapsto\left(x^{m}, y\right)$ for some integer $m \geqslant 2$. In particular, $g^{-1} \mathscr{I}_{p_{i}} \cdot \mathscr{O}_{M, q_{i}}=\left(x^{m}, y\right) \mathscr{O}_{M, q_{i}}$. A straightforward computation shows that $(g \circ \mu)^{-1} \mathscr{I}_{p_{i}} \cdot \mathscr{O}_{S}$ is not invertible, yielding a contradiction. Thus $p_{i} \notin R_{3} \backslash \operatorname{Supp}\left(R_{2}+R_{4}\right)$. Similarly, $p_{i} \notin R_{4} \backslash \operatorname{Supp}\left(R_{1}+R_{3}\right)$. This proves the claim. Notice that $\# g^{-1}\left(p_{i}\right)=1$ if $p_{i} \in R_{3} \cap R_{4}$, while $\# g^{-1}\left(p_{i}\right)=m^{2}$ if $p_{i} \in N \backslash \operatorname{Supp}(g(R))$. We have

$$
\sum_{1 \leqslant i \leqslant k} \# g^{-1}\left(p_{i}\right)=K_{M}^{2}-K_{S}^{2}=7-K_{S}^{2} \leqslant 6 .
$$

Observe that $\# g^{-1}\left(p_{i_{0}}\right) \geqslant 2$ for some $1 \leqslant i_{0} \leqslant k$ since $K_{S}^{2}<K_{T}^{2}$. It follows from the above discussion that $p_{i_{0}} \in N \backslash \operatorname{Supp}(g(R))$. We conclude that $m=$ 2. Let $C$ be the strict transform in $N$ of a ruling of $\mathbb{P}^{1} \times \mathbb{P}^{1}$ passing through $\varepsilon\left(p_{i_{0}}\right)$. Then $g^{-1}(C)$ is the union of 2 disjoint smooth rational curves with zero self intersection, and $\mu$ blows up 4 points on $\operatorname{Supp}\left(g^{-1}(C)\right)$, yielding a contradiction. This completes the proof of the theorem.

Example 10.7. Set $M=\mathbb{P}^{1} \times \mathbb{P}^{1}, N=\mathbb{P}^{2}$, and consider the double cover $g: M \rightarrow N$ induced by the projection $\mathbb{P}^{3} \rightarrow-\rightarrow \mathbb{P}^{2}$ from a general point. Let $T \simeq \mathbb{F}_{1}$ be the blow up of $N$ a general point $p, S$ the blow up of $M$ along $g^{-1}(p)$, and $f: S \rightarrow T$ the induced (finite) morphism. If we denote the exceptional curve of $\nu: T \rightarrow N$ by $F$, then an easy computation gives $\mathscr{O}_{S}\left(-K_{S}\right) \simeq$ 
$f^{*}\left(\nu^{*} \mathscr{O}_{\mathbb{P}^{2}}(2) \otimes \mathscr{O}_{S}(-F)\right)$, and therefore, $-K_{S}$ is ample. We have $K_{S}^{2}=6$ and $K_{T}^{2}=8$.

\section{Acknowledgements}

We are grateful to Cinzia CASAGRAnde for very fruitful discussions. We also thank the referee for their thoughtful suggestions on how to improve the presentation of some of the results.

\section{References}

[1] F. Ambro, The moduli b-divisor of an lc-trivial fibration, Compos. Math., 141 (2005), no. 2, 385-403.

[2] T. Ando, On extremal rays of the higher-dimensional varieties, Invent. Math., 81 (1985), no. 2, 347-357.

[3] M. Andreatta and J. A. Wiśniewski, A view on contractions of higherdimensional varieties, in Algebraic geometry-Santa Cruz 1995, Vol. 62 of Proc. Sympos. Pure Math., 153-183, Amer. Math. Soc., Providence, RI (1997).

[4] A. Beauville, Endomorphisms of hypersurfaces and other manifolds, Internat. Math. Res. Notices, (2001), no. 1, 53-58.

[5] L. Bonavero, C. Casagrande, O. Debarre, and S. Druel, Sur une conjecture de Mukai, Comment. Math. Helv., 78 (2003), no. 3, 601-626.

[6] F. Campana, Connexité rationnelle des variétés de Fano, Ann. Sci. École Norm. Sup. (4), 25 (1992), no. 5, 539-545.

[7] C. Casagrande, Quasi-elementary contractions of Fano manifolds, Compos. Math., 144 (2008), no. 6, 1429-1460.

[8] F. R. Cossec and I. V. Dolgachev, Enriques surfaces. I, Vol. 76 of Progress in Mathematics, Birkhäuser Boston, Inc., Boston, MA (1989), ISBN 0-8176-3417-7.

[9] I. V. Dolgachev, Classical algebraic geometry, Cambridge University Press, Cambridge (2012), ISBN 978-1-107-01765-8. A modern view.

[10] O. Fujino, Fundamental theorems for the log minimal model program, Publ. Res. Inst. Math. Sci., 47 (2011), no. 3, 727-789. 
[11] O. Fujino and Y. Gongyo, On canonical bundle formulas and subadjunctions, Michigan Math. J., 61 (2012), no. 2, 255-264.

[12] O. Fujino and H. Sato, Smooth projective toric varieties whose nontrivial nef line bundles are big, Proc. Japan Acad. Ser. A Math. Sci., 85 (2009), no. 7, 89-94.

[13] M. Fulger, The cones of effective cycles on projective bundles over curves, Math. Z., 269 (2011), no. 1-2, 449-459.

[14] T. Graber, J. Harris, and J. Starr, Families of rationally connected varieties, J. Amer. Math. Soc., 16 (2003), no. 1, 57-67 (electronic).

[15] A. Grothendieck, Cohomologie locale des faisceaux cohérents et théorèmes de Lefschetz locaux et globaux (SGA 2), Documents Mathématiques (Paris) [Mathematical Documents (Paris)], 4, Société Mathématique de France, Paris (2005), ISBN 2-85629-169-4. Séminaire de Géométrie Algébrique du Bois Marie, 1962, Augmenté d'un exposé de Michèle Raynaud. [With an exposé by Michèle Raynaud], With a preface and edited by Yves Laszlo, Revised reprint of the 1968 French original.

[16] P. Jahnke and I. Radloff, Gorenstein Fano threefolds with base points in the anticanonical system, Compos. Math., 142 (2006), no. 2, 422-432.

[17] Y. Kawamata, K. Matsuda, and K. Matsuki, Introduction to the minimal model problem, in Algebraic geometry, Sendai, 1985, Vol. 10 of Adv. Stud. Pure Math., 283-360, North-Holland, Amsterdam (1987).

[18] J. Kollár, Y. Miyaoka, and S. Mori, Rational connectedness and boundedness of Fano manifolds, J. Differential Geom., 36 (1992), no. 3, 765779 .

[19] J. Kollár and S. Mori, Birational geometry of algebraic varieties, Vol. 134 of Cambridge Tracts in Mathematics, Cambridge University Press, Cambridge (1998), ISBN 0-521-63277-3. With the collaboration of C. H. Clemens and A. Corti, Translated from the 1998 Japanese original.

[20] R. Lazarsfeld, A Barth-type theorem for branched coverings of projective space, Math. Ann., 249 (1980), no. 2, 153-162.

[21] Q. Li, Pseudo-effective and nef cones on spherical varieties, Math. Z., 280 (2015), no. 3-4, 945-979. 
[22] S. Mori and S. Mukai, Classification of Fano 3-folds with $B_{2} \geq 2$, Manuscripta Math., 36 (1981/82), no. 2, 147-162.

[23] M. V. Nori, Zariski's conjecture and related problems, Ann. Sci. École Norm. Sup. (4), 16 (1983), no. 2, 305-344.

[24] G. Occhetta, L. E. Solá Conde, K. Watanabe, and J. A. Wiśniewski, Fano manifolds whose elementary contractions are smooth $\mathbb{P}^{1}$-fibrations (2014). Preprint, arXiv:1407.3658.

[25] V. V. Przhiyalkovskiŭ, I. A. Chel'tsov, and K. A. Shramov, Hyperelliptic and trigonal Fano threefolds, Izv. Ross. Akad. Nauk Ser. Mat., 69 (2005), no. 2, 145-204.

[26] K. Watanabe, Certain invariant subrings are Gorenstein. I, II, Osaka J. Math., 11 (1974) 1-8; ibid. 11 (1974), 379-388.

[27] J. A. Wiśniewski, On contractions of extremal rays of Fano manifolds, J. Reine Angew. Math., 417 (1991), 141-157.

Institut Fourier, UMR 5582 du CNRS, Université Grenoble Alpes CS 40700, 38058 Grenoble Cedex 9, France

E-mail address: stephane.druel@univ-grenoble-alpes.fr

Received December 31, 2014 